\title{
An Egocentric Semantic Reference System for Affordances
}

Editor(s): Aldo Gangemi, Institute of Cognitive Sciences and Technologies, Italy

Solicited review(s): Giovanni Pezzulo, Institute of Cognitive Sciences and Technologies, Italy; Gaurav Sinha, Ohio University, USA; Aldo

Gangemi, Institute of Cognitive Sciences and Technologies, Italy

\author{
Jens Ortmann ${ }^{\mathrm{a}, *, * *}$, Giorgio De Felice ${ }^{\mathrm{b}}$, Dong Wang ${ }^{\mathrm{c}}$ and Desiree Daniel ${ }^{\mathrm{c}}$

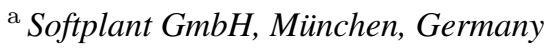 \\ Email: jens.ortmann@softplant.de \\ ${ }^{\mathrm{b}}$ Department of Mathematics and Informatics \\ University of Bremen, Germany \\ E-mail:defelice@informatik.uni-bremen.de \\ ${ }^{\mathrm{c}}$ Institute for Geoinformatics (IfGI) \\ University of Münster, Germany \\ E-mail: \{wang.dong,desiree.daniel\}@uni-muenster.de
}

\begin{abstract}
This article suggests a theory of egocentric semantic reference systems for human observations of affordances that can be used to semantically account for subjective human observations on the web. Based on the perceptual theory of affordances, which suggests that humans perceive the potential actions the environment affords, an egocentric semantic reference frame is established, which is anchored in the observer's specific capabilities for perception and action. The theory is completed with transformations that allow to project values of observed affordances from one user's ordinal reference frame into another user's ordinal reference frame. The potency of the theory to capture the semantics of human observations is demonstrated through the implementation of a full fledged egocentric semantic reference system for the prototypical affordance of hikability that a mountain path affords to a hiker. The prototype uses real user-ratings from a community driven web portal.
\end{abstract}

Keywords: Affordance, Egocentric Reference System, Human Observation, Semantic Reference System

...man is "the measure of all things, of the existence of the things that are and the non-existence of the things that are not." [Protagoras as quoted in $30,152 \mathrm{a}]$

\footnotetext{
*Corresponding author. E-mail: jens.ortmann@ softplant.de.

${ }^{* *}$ This work has been partly supported through the International Research Training Group on Semantic Integration of Geospatial Information by the DFG (German Research Foundation), GRK 1498 and by the China Scholarship Council.
}

\section{Introduction}

The above quote, which is attributed to the Greek philosopher Protagoras (490-420 BC) ${ }^{1}$ has not lost any of its original significance and controversy. In particular in the field of observations and measurements the reference to the observer is an important, but often neglected aspect. Motivated by the opportunities and promises of the semantic web, this paper suggests a theory of egocentric semantic reference systems for human observations of affordances, which uses an on-

${ }^{1}$ see http://en.wikipedia.org/wiki/Protagoras (all websites in this article were accessed 27.01.2012) 
tology as semantic reference frame. We show how this theory can overcome the problems and inaccuracies caused by a questionable objectivism, which assumes human-independent observations and measurements. Current approaches to represent human observation fall short of representing the subjectivity of observations and often perform computations, such as averaging, that are not possible on ordinal reference frames typically used by human observers. For example, what a Dutch tourist perceives as a "challenging hike" might be "a stroll" for a Swiss mountaineer and what the Swiss mountaineer perceives as a "challenging hike" might actually put the Dutch tourist in serious danger.

Egocentric reference systems have been well established in Geographic Information Science. Most people are used to egocentric reference frames from car navigation systems that adopt the driver's perspective. Egocentric reference frames are often contrasted with allocentric reference frames that use an external reference, for example the equator as reference for latitude values of geographic coordinates. In this paper we extend the distinction between egocentric and allocentric reference systems from geographic to semantic reference systems. A semantic reference system allows to assign symbols to any observable instead of only coordinates to locations on earth.

This work adopts the theory of affordances [14,15], to explain how and what humans perceive. The theory of affordances suggests that humans perceive opportunities for action. These opportunities for action are always taken with reference to the observer. Hence, human observations are inherently subjective. The perception of opportunities for action fits well the many social web platforms, where users can rate amenities, services and opportunities for activities. The affordance theory is combined with accounts of human judgements in computer science $[7,9,8]$ and introduced to previous work on reference systems [22,23,32,33]. It is hypothesized that an egocentric semantic reference system for human observations of affordances allows capturing the subjective meaning of observation values in ordinal reference frames. Thereby, an egocentric semantic reference system establishes the individual observer as the measure of her own observations, while at the same time making the subjective observation values usable in information systems and social web applications. Semantic transformations across individual reference frames will enable a transformation of values from one person's reference frame into another person's reference frame. We validate the theory of egocentric semantic reference system by provid- ing a full implementation of a semantic reference system. To the author's knowledge this is also the first implementation of a real semantic reference system with all its components.

The next section presents the background on reference system, affordances and human observation in information system. Section 3 introduces the two leading examples of stair-climbability and mountainhikability. In Section 4 and 5 the theory of a semantic reference system with datum, reference frame and transformations is presented and afterwards prototypically implemented as described in Section 6. Section 7 discusses the achievements and limitations of this work, before concluding in Section 8.

\section{Background and Related Work}

This section presents the central ideas of reference systems, affordances and human observations, which we use to establish egocentric semantic reference systems for observations of affordances.

\subsection{Reference Systems}

Reference systems are fundamental to information [31,5,22]. A reference system basically establishes a set of shared reference entities (usually something observable) to allow describing new things in terms of the reference entities. Reference systems emerged from the need to compare observations and measurements. One of the first to investigate reference systems on a general level was Stanley Stevens, who suggests a very basic definition of measurement as "the assignment of numbers to objects according to a rule" [43, p. 677 ; as quoted in [5]]. A reference system specifies these rules. Chrisman [5] distinguishes reference systems for space, time and attribute as the three types of reference systems for geographic information. Kuhn [22] and Kuhn and Raubal [23] generalize from these types of reference systems to a theory of semantic reference systems.

\subsubsection{Spatial Reference Systems}

In a spatial reference system, the position of a point is identified by two or three dimensional coordinates. In a geographic reference system, a spatial reference system for the earth, the coordinates are assigned to places on earth. These coordinates are distributed on a virtual grid and establish the reference frame. Some fix-points (reference points) in the reference frame are 


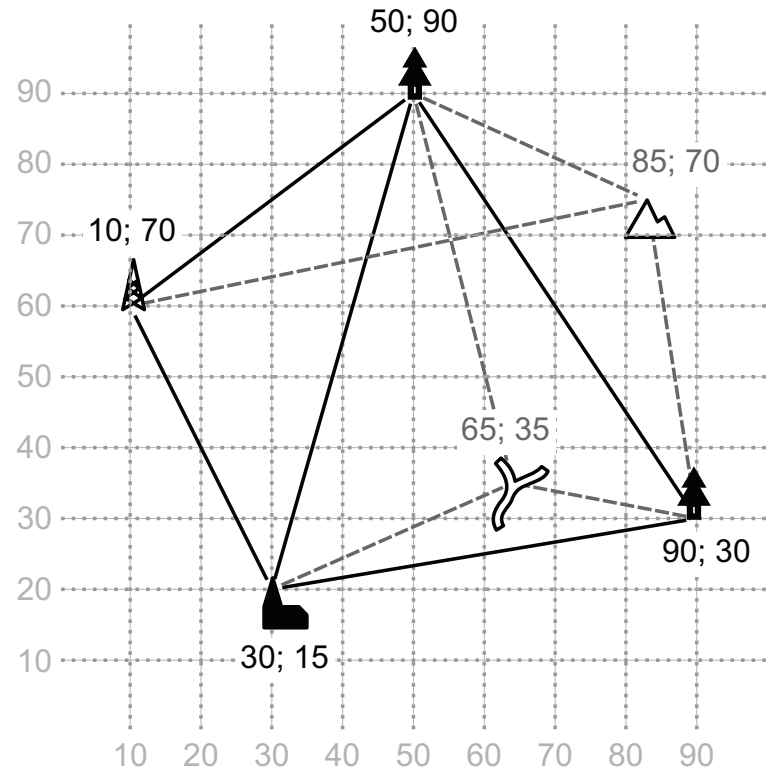

Fig. 1. Schematic representation of a spatial reference system. The reference frame (dotted grid) is anchored in landmarks whose coordinates are known (black coordinates). New places like the mountain peak or the road crossing get a coordinated (grey) based on the distance to place whose coordinates are known.

tied to physical, observable points by explicitly assigning that this point in the reference frame represents this physical location on earth. A regional reference frame is often realized as a Cartesian coordinate system. Global reference systems use angular distances and their reference frame is realized as a polar coordinate system. Figure 1 illustrates how a reference frame defines a virtual grid that assigns coordinates to places.

Additionally, a reference frame has a map scale given by the ratio of distance in the representation and distance in the real world. The map scale usually determines the level of detail displayed. An ontology design pattern to describe the map scale and to integrate different information sources under explicit consideration of the map scale is presented in [4].

The reference points realize the datum. For example, the World Geodetic System 84 (WGS84) $^{2}$ uses angular distances from the equator (latitude) and from the meridian that runs through Greenwich, UK (longitude). The reference entities can be chosen arbitrarily. The choice of the zero meridian of, for instance the WGS84, does not influence the accuracy and uniqueness of the reference system. However, to facilitate re-

\footnotetext{
${ }^{2}$ See http://en.wikipedia.org/wiki/World_ Geodetic_system for more information.
}

producibility a famous landmark (the royal observatory in London) was originally chosen to fix the zero meridian. In this case, the great circles and the meridians form an ellipsoid that approximates the shape of the earth. The ellipsoid serves as reference surface for the coordinate system. It is anchored in a set of known points. The ellipsoid can be described by a set of parameters, such as length of major axis, flattening and parameters for the orientation relative to the earth. These parameters specify the datum of the reference frame [5]. In Figure 1 the places denoted by black coordinates show the fix points that anchor the reference frame. The grey coordinates are assigned to places by establishing the distance relation between the place in question and places whose coordinates are already known.

Depending on how a datum is specified, different types of datums can be distinguished. Relevant for this work are allocentric and egocentric datums. An allocentric datum is defined independently of an agent and it does not depend on the choice of reference points [21]. Most spatial datums that are used in Geographic Information Systems are allocentric. An egocentric datum models the perspective of one particular agent [21]. A car navigation system that gives direction of right and left turns from the driver's perspective can be seen to adopt the egocentric spatial datum of the driver. The reference frame is solely anchored in the location and orientation of the car driver and the reference frame moves as the car moves. A bridge that is "in front of" the car will be "behind" the car after driving over the bridge and whether you have to turn left or right to reach the city center depends on the direction you are coming from.

The third component of a reference system is composed by transformations that allow an integration of values across different reference frames. For example, one might want to convert the coordinates given in a regional reference frame such as Gauss-Krüger ${ }^{3}$ into the global reference frame of the WGS84 using a polar coordinate transformation. Transformations are important when integrating datasets, for example across different countries which use their individual national reference systems.

\subsubsection{Semantic Reference Systems}

The commonalities of spatial, temporal and attribute reference systems led Kuhn [22] to suggest semantic

\footnotetext{
${ }^{3}$ the Gauss-Krüger coordinate system is a regional reference system used for instance in Germany.
} 
reference systems as a generalization of the known reference systems. A semantic reference system is not restricted to assigning numbers to objects, but allows assigning any symbol to an object, thereby allowing to account for the semantics of the symbol.

Kuhn and Raubal [23] and Janowicz and Scheider [19] suggest implementing reference frames for semantic reference systems with ontologies. The semantic reference frame specifies a set of allowed symbols. Probst [32,33] investigates semantic reference frames for observations and measurements grounded in the Descriptive Ontology for Linguistic and Cognitive Engineering (DOLCE) [26]. Probst provides an ontological theory for unary qualities and an implementation in the Web Ontology Language (OWL) ${ }^{4}$. To overcome the agent-independence of Probst's approach, Ortmann and Daniel [27] suggest an ontology design pattern for referential qualities that can be used to model qualities that depend on their host and on an additional referent entity. The ontology design pattern for referential qualities uses the same version of DOLCE as Probst's work $[32,33]$.

The datum in a reference system anchors the reference frame. It fixes the interpretations of the primitive terms and values in the reference frame. Fixing the interpretation requires a reference to the real world. The reference, which points from the system of symbols into the real world, is a foundational problem of reference systems. It has been dubbed the 'symbol grounding problem' [16]. Two approaches have been proposed for semantic reference systems to deal with this problem. Firstly, one can simply assume the meaning of a fixed set of symbols to be given, for example by a foundational ontology [23]. A foundational ontology defines the general and shared top-level notions. Secondly, the meaning of symbols denoting observable entities can be grounded in perceptual operations that are shared across humans and that can be specified in information systems $[40,41,39]$. In either case, the meaning of new symbols can then be defined in terms of the existing symbols, thereby restricting the interpretation of the new symbols within the reference frame. Figure 2 provides a schema that shows how a semantic reference frame generalizes a spatial reference frame to reference observables of any type. Especially, the similar network structures of nodes that have values and edges that specify the relations between the nodes are shown in Figure 1 and 2.

\footnotetext{
${ }^{4}$ see http://www.w3.org/TR/owl-features/
}

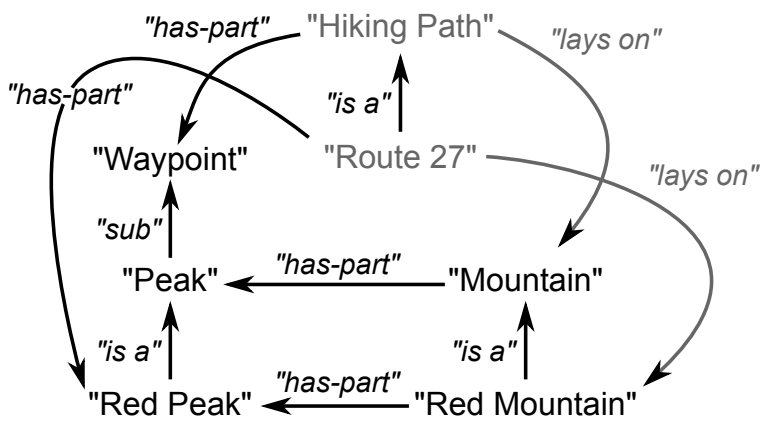

Fig. 2. Schematic representation of a simple semantic reference frame. Similar to a spatial reference frame, observables are assigned values. The values in a semantic reference frame are labels. From a given set of values, for instance waypoint, peak and mountain, it is possible to reference a new value for a hiking path and relate it to the known values.

The distinction of allocentric and egocentric datums and reference frames has not yet been investigated for semantic reference systems. However, to the authors knowledge all currently existing semantic reference frames for observations and measurements are allocentric. A closer examination of affordances in the next subsection will indicate that the explicit inclusion of the observing agent as a referent for its observations leads to egocentric reference frames for affordances, which take the personal perception and expression of the observer as set of fixed terms.

Transformations across semantic reference frames are the least explored component of semantic reference systems. In Geographic Information Science, Schade [38] offers an approach to transform schemata for geographic data sets encoded in the Web Service Modelling Language (WSML) ${ }^{5}$ using the functional language Haskell.

\subsection{Affordances}

Gibson $[14,15]$ devises a theory of how animals ${ }^{6}$ perceive opportunities for action. These opportunities for action are called affordances. Gibson introduces the notion of affordance as follows:

The affordances of the environment are what it offers the animal, what it provides or furnishes, whether for good or ill. The verb to afford is found

\footnotetext{
${ }^{5}$ see http: / / www.w3.org/Submission/WSML/

${ }^{6}$ In this work the term "agent" is used as the more general term, encompassing humans and animals. Gibson studied animals and humans, and most ecological psychologists study humans. When referring to specific work the respective types of agents are used.
} 
in the dictionary, but the noun affordance is not. I have made it up. I mean by it something that refers to both the environment and the animal in a way that no existing term does. It implies the complementarity of the animal and the environment. [15, p. 127 , emphasis in original]

Even more important for our program, Gibson states that

affordances are properties of things taken with reference to the observer. $[15$, p. 137 , emphasis in original]

Gibson characterizes an affordance as an observable property of a thing that an animal can act upon. Furthermore, Gibson establishes the perceiving animal as the measure of all the things it observes.

Despite the intuitiveness and appeal of the idea of affordances, there exists no single ontology of affordances. Since Gibson was not able to fully develop the theory of affordances [20], there exist competing views on how the notion should be defined. We follow Shaw et al. [42], who suggested that affordances are properties of the environment that are complemented by socalled effectivities, which are properties of the animal. Turvey [45] formalized this dual model of affordances and thereby provided the first formal specification of what an affordance is. ${ }^{\text {? }}$

Warren [46] provides an early experimental proof of the human ability to perceive affordances. His experiments show that humans can judge the climbability of stairs nearly perfectly, and that this ability can be modelled as depending on the observing human's leg length, which is the reference for the riser height of the stairs. Similar experiments have been conducted for example, for reachability [3], sitability of chairs [24] or crossability of a road [29]. Generally, these experiments provide evidence that humans can accurately perceive which actions they can perform and how difficult these action are to them. In the cited experiments the humans were asked to express their perceptions and the degree to which the affordance was provided to them (except for [29] where the actual actions were observed).

Ortmann and Kuhn [28] investigate the place of affordances in an information system ontology of observations. Following the interpretation of affordances

\footnotetext{
${ }^{7}$ Stoffregen [44] suggests an alternative formal specification where affordances are relational system-properties at the boundary of animal and environment.
}

suggested by Shaw et al. [42] and Turvey [45], Ortmann and Kuhn [28] model affordances as qualities in DOLCE. DOLCE characterizes qualities "as the basic entities we can perceive or measure" [26, p. 16]. Ortmann and Daniel [27] refine the view of affordances as qualities by making the observing agent as a referent explicit. In their view an affordance is a referential quality, which is specifically dependent on its host and on the observer.

\subsection{Representing Human Judgements}

Freksa [7,8] and Freksa and López de Mántaras [9] investigate human observations and their representation in information systems in comparison to metric measurements. Measurements are well established in the hard-sciences, where scientists mostly deal with metric measurements, but in the soft-sciences the scientists often face descriptive observations and judgements made by human subjects. Experiments conducted by Freksa and López de Mántaras [9] and Freksa [8] suggest that human judgements of phenomena should be represented through verbal descriptions rather than metric values. The verbal descriptions refer to ordered categories that are represented as simplified version of fuzzy sets [48]. Therefore, instead of making the judgements appear more precise through translating them into a metric system, the imprecision is preserved, but made explicit. Hence, more information about the actual judgement is preserved. Freksa [7] argues that this approach requires only comparatively small vocabularies of judgements, but is still more accurate than metric human judgements. A small set has the advantage that it is rather easy to handle, yet its expressiveness and granularity might be limited. However, experiments show that the results of human judgements using even small sets of descriptions are superior in accuracy and expressiveness to the results of the same humans using numeric values, if the fuzziness of the judgements is made explicit $[9,8]$.

Freksa [7] suggests two types of context-dependent adaptation for the semantics of the terms used as judgements. The concept of global adaptation refers to the problem that the same set of values (terms) can be used differently. For example, judgement of distance is different whether you talk about your travel destination or your workout run. Local adaptation refers to different judgements based on a set of given objects of the same type. Encountering three trees with heights $4 \mathrm{~m}, 6 \mathrm{~m}$, and $8 \mathrm{~m}$, an observer would refer to the latter tree as "tall", yet if there are three trees $8 \mathrm{~m}, 10 \mathrm{~m}$ and 
$12 \mathrm{~m}$, then the same observer would likely refer to an equally high tree as "small".

Such subjective and context-dependent judgements can be made more objective when there is a reference object. In the stair-climbing experiment it is possible to objectivize the subjective judgements through the measurement of the leg length of the observing person, but this is not possible in the case of user-ratings, where every user has its own (local) set of ratings.

Aiming for a mathematical model of human observation Zadeh [49,50,51] promotes a computational theory of perception. One of his observation is that

perceptions are f-granular, meaning that (1) the boundaries of perceived classes are unsharp and (2) the values of attributes are granulated, with a granule being a clump of values (points, objects) drawn together [...]." [49, p. 73]

In contrast to this, measurements are numerical and crisp. In a similar way to Freksa [7,8] and Freksa and López de Mántaras [9], Zadeh [49,50,51] first argues for a distinct treatment of perceptions based on their specific characteristics, but goes on to state that the computational theory of perception applies to both, perceptions and measurements, or in other words, that measurements are a special case of perception. In essence, the computational theory of perception takes linguistic propositions and assigns fuzzy semantic constraints to them. The computational theory offers means to compute and propagate the fuzzy truth values.

The presented work imparts the important insights that (1) the results of (technical) measurements and (human) perception fall in different scales, but that (2) there exists a theory of perception that is general enough to cover the results of measurements and perception.

\section{Examples}

Two exemplary affordances are used to show the potency of egocentric semantic reference systems for affordances: (1) The stair-climbability afforded by a step to a person and (2) the hikability afforded by a hiking path to a hiker. The examples are chosen from very different domains and scales each capable of demonstrating the specific peculiarities and challenges that occur at that scale and domain. Additionally, this allows highlighting the specific characteristics of very different domains and shows that our approach is general enough to deal with them without any modification.

\subsection{The Stair-Climbing Affordance}

The stair-climbing affordance is the affordance that is offered by a step to a person to climb up that step. It can be easily extended to other objects like the affordance to climb on a chair. The stair-climbing affordance has been investigated in laboratory conditions by the psychologist William H. Warren [46]. It has become a standard example in Ecological Psychology.

The practical relevance of investigating and documenting simple and daily affordances such as the stairclimbability affordance lies, for example, in the design of navigation applications for elderly or handicapped people and in the design of ambient assisted living environments. ${ }^{8}$ The greater difficulties that elderly people have in climbing stairs or reaching out to a cupboard have to be taken into consideration to design conveniently liveable environments.

Warren [46] conducted a set of experiments with a group of his students as his subjects. Warren used pictures of stairs instead of real stairs and asked his students to judge the climbability. His results show that his students are perfectly capable to judge the least climbable stairs (i.e. the maximum riser height that the subject thought she or he could climb) and also the optimally climbable stairs (i.e. the stairs with a riser height that appeared most convenient for them to climb). The results suggest two things. Firstly, the perception of his subjects is in perfect accordance to clinical studies and measurements of bodily capabilities and secondly, the riser height of the stairs chosen for least or optimal climbability depends on the subjects leg length. Warren even identifies a ratio between leg length and riser height that is the same across all his subjects. The homogeneity of the group of subjects with respect to their physical fitness and abilities allows for modelling the affordance of stair-climbability based on only one parameter. A more heterogeneous group of people and a more diverse set of objects would require more parameters to accurately model the stair-climbability.

The experiments show that humans have a reference system for stair climbability according to which they can judge the climbability of stairs upon perception. In Warren's [46] set up, the reference frame for climbability can be anchored in the subject's leg length.

\footnotetext{
${ }^{8}$ Ambient assisted living environments are environments that are equipped with non-intrusive technology to support elderly people in independent daily life in their familiar surroundings.
} 


\subsection{The Hikability Affordance of a Mountain Path}

The hikability affordance is the affordance to hike that a mountain path offers to a hiker. This example is inspired by a web portal ${ }^{9}$ where users can share their reports and judgements of hiking tours. Similar portals exist for many amenities and activities such as hotels, restaurants, running-, skating- and cycling tracks, playgrounds etc. The ratings of the degree to which an affordance is offered to a person is usually made after acting on the affordance.

The individual rating of an amenity cannot be modelled through physical parameters as it is possible for the stair-climbing affordance. Indeed, the hikability of a mountain path depends on the hiker's head of heights, surefootedness, stamina, ability to cope with thin air and on the mountain path's steepness, exposure, height and on many other characteristics. Furthermore, it is not always clear how the properties of the mountain paths are to be taken with reference to the capabilities of the hiker. When rating hotels and restaurants, parameters for social and cultural aspects have to be added. In current rating portals, the rating of a service, amenity or activity is simply the average of all users. However, as indicated before this ignores a bias in the group of observers. For example, a hotel in an area with a lot of nightlife is rated by the predominantly young guests, the high rating might not apply to a family and their expectations. The same applies to the hiking example. A route in exposed and rocky terrain might be rated in a medium difficulty by hikers used to exposed routes, but regardless of the fitness, a person from the lowlands might find the tour very difficult.

To model egocentric reference systems for the hikability affordance that a mountain path offers to a hiker, we have crawled data from the online platform hikr.org. Hikr.org is a community driven portal where registered users can share tour reports. The tour reports have a name, date and contributing hiker, and can have additional attributes such as a list of waypoints (peaks, huts and other landmarks), duration, height gain, height loss, and most importantly a rating. There exist different ratings for hiking paths, winter tours, climbing routes etc. For this work, only the hiking paths and their hikability ratings are of interest. The hiking rating is given according to a scheme ranging from T1 ("Valley Hike") to T6 ("Difficult Alpine

\footnotetext{
${ }^{9}$ http://www.hikr.org
}

Hike"). Tours from all over the world have been contributed, but most tours are from Europe, with a particular bias to Switzerland. Therefore, only tours from Switzerland are taken into account for the prototype. The earliest tour crawled is from 2006 and the extracted dataset ranges until November 6th, 2011.

From the website, a total of 22913 tours were crawled, 15956 of them have a rating. These tours were contributed by 1152 users. The contributions made per user follow a power law distribution, with the topcontributor having 668 tour reports, 81 users having more than 100 reports and a long tail of 313 users having only one report. The tours have a total of 14042 waypoints assigned to them. The waypoints can appear in several tour reports, for example, the waypoint for the Matterhorn appears in 21 tour reports.

\section{Datums and Reference Frame for Affordances}

In this section the datum and the reference frame of an egocentric reference system for affordances are introduced. These two aspects are individual to the user and manifest the 'man as measure' postulation. The semantic datum specifies how the primitive values are related to the observable world. The semantic reference frame specifies how the values are related to each other and how values can be derived from the primitive ones. This section develops generally applicable statements about egocentric datums and reference frames for affordances. Readers familiar with foundational ontologies will notice that the terminology used here is inspired by DOLCE. This is not necessary for the theory but is a result of previous work [e.g. 32,33,28,27] and the intention to make this theory applicable in semantic web applications.

\subsection{The Egocentric Datum}

The semantic datum specifies how observations values are related to the observables in the world. The semantic datum for affordances specifies how the terms of one particular observer refer to the affordances she observes in the world. Akin to the theory of affordances [15,17], the specification of the semantic datum for affordances makes the observing agent explicit. It takes the observing agent with her qualities and capabilities as the yardstick for the observations. This idea of using the observing agent as a measure for its perception is found throughout the affordance literature [e.g. 15,17,45]. We assume the observer expresses her 


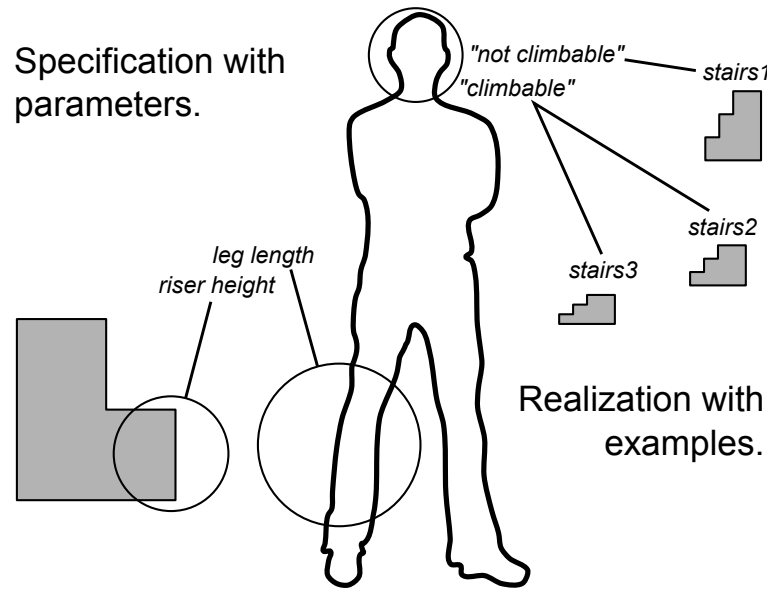

Fig. 3. The observer provides the datum for the reference frame. In the information system the datum can be specified through parameters or realized through a set of fixed sample observations.

observation and thereby provides a set of initial values for observed affordances. This initial set of values, whose meaning is clear to the observer, suffices to specify the semantic datum of the observer. Bringing the theory of affordances into the research on datums in semantic reference systems leads to egocentric semantic reference systems. In an egocentric reference system the human observer is modelled as part of the reference system in the information system. Hence, a partial representation of the user is necessary, for example by specifying qualities or capabilities of the user or by explicitly relating the results of observations to the observer.

In general, we distinguish two ways to formalize the semantic datum in an information system. Firstly, the egocentric datum can be specified through a set of parameters that represent qualities or capabilities of the observer taken with reference to an observable object that provides the affordance. Secondly, the egocentric datum can be realized through a set of reference observables that the user has expressed. The difference between specifying and realizing a semantic datum is similar to the difference between an intensional and an extensional definition of a set. The specification uses parameters to define a mapping between the observables to the values. The realization uses an explicit set of values that are related to an explicit set of observables. Specification and realization of the datum are illustrated in Figure 3.

In the example of the stair-climbing affordance Warren specifies a datum with one parameter that is sufficient to approximate the person's judgements. The pa- rameter is the observer's leg length. The person's leg length is different but fixed for every observer. Hence, the egocentric semantic datum for affordances is determined by the observer and has individual but fixed parameters. The knowledge about the datum parameters allows specifying the perceptual operations, which ground the meaning of expressions [41,39].

Unfortunately, the parameters that specify the semantic datum are not always known. The observer usually does not make the datum explicit. The datums for observations of affordances that are more complex than the stair-climbing affordances and that cannot be modelled based on very few easily measurable physical parameters cannot be specified in this way. For example, the hikability affordance does not only depend on one property of the observer, but a whole set of properties has to be considered, probably with a different individual weighting. In this case, only the realization of the datum through a set of reference points that anchor the reference frame can be derived from the observer's expressions and judgements.

Every individually observed affordance of the same type that is expressed by the observer explicates one particular fix-point that realizes the egocentric datum. For example, the set of rated reports that a hiker posts online realizes the user's semantic datum for hikability in the information system. ${ }^{10}$

The statements about the egocentric semantic datum make an important assumption about the symbol grounding problem [16]. The egocentric semantic datum for affordances does not explicitly deal with the symbol grounding problem, but it can be seen to embrace the problem by assuming that there exists an environment that is shared by observers, and that the observers have equivalent access to.

For the egocentric datum this means that the parameters of an egocentric datum are given in other reference frames that are grounded in their respective datum. For example, the leg length and riser height of the stair-climbability datum require a reference system for length and height measurement. Such reference systems exists and are well known and used. Therefore the grounding problem of stair-climbability is shifted to the grounding problem of length and height and thus excluded from this work. ${ }^{11}$

\footnotetext{
${ }^{10}$ The rating can change according to seasonal or weather conditions, which are not considered in this work.

${ }^{11}$ How to deal with the grounding problem has been scrutinized by Scheider [39].
} 
For the realization of the datum through shared observations it is assumed that the observer performs the same observation procedure for each individual hikability affordance. Additionally, it is assumed that observers can identify mountain paths and that a mountain path rated by one person can also be hiked and rated by another person with her own egocentric datum. This corroborates Gibson's claim of a shared environment across observers [15] and avoids solipsist positions that could otherwise be derived from egocentric reference systems. Hence, the perceived affordance (its value) is specific to the observer, but the environment, which provides the affordances, serves as a common ground for all observers.

The benefit of evading some of the challenges in the semantic datum comes at the price of a stronger focus on semantic translations. Interoperability among egocentric semantic reference frames ultimately depends on semantic translations from one user's egocentric reference frame into another user's egocentric reference frame.

\subsection{The Egocentric Reference Frame}

The egocentric semantic reference frame for affordances specifies the scale and units for the magnitudes of an observed affordance. A magnitude can be seen as a mapping from degrees of an affordance into units of magnitude [2]. A scale is a mode of representation that entails the order and the spacing of the magnitude units that represent the degrees of the observable affordance [2]. In the following the magnitude units are called values. If the semantic datum that anchors the reference frame is realized, some of the values are explicitly linked to representations of observables. Additionally, the reference frame allows to assign values to observables, which are not yet explicitly rated by the user. The datum can be seen to span the reference frame, and the reference frame specifies how the spanned space is filled with values.

The values do not need to have the same extent on the scale, the values can be fuzzy and they might overlap. Theoretically, each observer can freely choose the values she uses to describe her observations. Practically, the range of values is usually very limited $[9,8]$. In the mentioned social web applications where users contribute their observations of, for example, the quality of a hotel or restaurant or the difficulty of a hiking path, the values are usually fixed and only few values are used.
Requiring a definite choice of only one rating per observation excludes fuzzy ratings from the application. For hotel ratings, portals often provide a reference frame ranging from zero stars (lowest) to five stars (highest) alluding to allocentric reference frames applied by hotelier associations. In the case of the hiking example a reference frame of values ranging from $\mathrm{T} 1$ ("Valley Hike") to T6 ("Difficult Alpine Hike") is applied. Naturally, the values of human observations can denote overlapping magnitudes and the values can be fuzzy, but the fuzziness is often excluded by offering a non-fuzzy set of predefined categories.

Predefined values might suggest that they are objective. Yet, the effects of local adaptation [7], when ratings are made in the context of previous experiences, make judgements subjective. Every observer has his own subjective mapping of observed degrees of an affordance to the values for his ratings. Every new rating is adapted to the previous ones. For example, the rating of the same hiking path by a skilled mountaineer and by a visitor on vacation might result in different experiences of difficulty and in different values, even though the same labels and descriptions of the values are used.

The scale of the reference frame specifies the relations among the values. For example, an ordinal scale defines an order among values and specifies an ordinal reference frame. Additionally, the relative extent of the magnitudes that the values refer to can be specified. For example an interval scale has values that are ordered and all values denote magnitude degrees of the same extent. The values of an egocentric semantic reference frame for affordances are usually of different size and denote categories. The judgements are usually ordered, but categories can overlap so that the order only applies to categories that do not overlap.

Figure 4, shows a reference frame for the affordance of stair-climbability. The values are delimited according to the results of the maximal and optimal climabability of stairs [46]. In this case, four values termed "low", "perfect", "high" and "too high" (as depicted in Figure 4) can be assumed and are laid out on an ordinal scale. "Perfect" is the value that is used to denote the stairs that are perfectly climbable. This value represents an atomic granule of the observer's perception. Stairs lower than perfectly climbable steps fall into the value "low", higher stairs belong to the value "high", unless the value of the riser height/leg length ratio exceeds the critical point. In a simplified case one can group the first three values into one value termed "climbable", where "climbable" = "low" or "perfect" 
or "high". The values in the reference frame have a different extent, from an atomic granule for "perfect" climbabiliy to a theoretically unlimited extent for steps that are "too high". The categories in this simple reference frame do not overlap, and the order relation holds across all categories.

In conclusion an egocentric reference frame specifies the allowed values and the relation among them. It usually consists of a set of descriptive values that are ordered on a nominal, ordinal or partly ordinal ${ }^{12}$ scale. The values represent sometimes fuzzy and overlapping sets of individual ratings. The ratings of individuals fall into one of the values of the reference frame.

The theory presented so far allows to establish egocentric semantic reference frames for affordances. The reference frames are grounded in egocentric semantic datums defined by the observing agents. Often a set of values can be predefined, but every user has her own interpretation of the categories that is manifested in the user's personal ratings. It is even possible to let every user have a personal set of units of any size and with labels that the user chooses. Thereby, the egocentric reference frame for affordances substantiates Protagoras' postulation of 'man as measure' in the context of observations.

\section{Transformations in Egocentric Semantic Reference Systems for Affordances}

Establishing egocentric reference systems at first undermines the idea of interoperability, which typically relies on a common basis. The grounding in a shared reference frame along with the transformation that can be built between egocentric reference frames of affordances turn affordances into operational observables. A subjective observations made by one user becomes a valuable information source for another user.

Borrowing from Geographic Information Science, two types of transformations are distinguished: (1) datum transformations and (2) direct transformations. A datum transformation is a transformation based on the datum parameters. The direct transformation establishes a transformation function based on observables, whose values are known in both reference frames.

\footnotetext{
${ }^{12}$ Partly ordinal in the sense that the order relation does not hold between overlapping values. This is not to be confused with a partial ordering.
}

\subsection{Datum Transformations}

The datum transformation requires explicit knowledge of the datum parameters. If all parameters are known for the input and the output reference frame, then a datum transformation can be derived.

For very simple, purely physical affordances the datum parameters are sometimes known and datum transformations can be derived. One such case is the stairclimbing affordance. In the following, one example of a datum transformation for stair-climbing reference frames (cf. Figure 4) is discussed. Despite the fact that the datum parameter leglength is given on a ratio scale, the datum transformation is a mapping between two ordinal reference frames.

One can define a transformation function $f$ that takes the parameter $p_{I}$ of the input datum, the parameter $p_{O}$ of the output datum and the value $v_{I}$ that should be transformed as input. The resulting transformation function is shown in Equation 1. To keep the example simple only the values "climbable" and "too high" are used in the equation. If the leg length parameter $p_{I}$ of the input datum is bigger than the leg length parameter $p_{O}$ of the output datum and the input value $v_{I}$ is "climbable", then the output value is "climbable" as well. If in the same case the value $v_{I}$ is "too high", then no constraint can be projected into the target reference frame and the output value can be "climbable" or "too high". If $p_{I}$ is smaller than $p_{O}$ and $v_{I}$ is "too high", then also the output value will be "too high". If in this case the rating is "climbable", again no statement can be made about the climabability and the output value is "climbable" or "too high".

$$
f\left(p_{I}, p_{O}, v_{I}\right)=\left\{\begin{array}{l}
\text { "climbable" } \\
\text { if } p_{I}>p_{O} \text { and } v_{I}=\text { "climbable" } \\
\text { too high" } \\
\text { if } p_{I}<p_{O} \text { and } v_{I}=\text { "too high" } \\
\text { "climbable" or "too high" } \\
\text { otherwise }
\end{array}\right.
$$

The transformation function with all four above mentioned values "low", "perfect", "high" and "too high" is shown in Equation 2. The transformations of ordinal reference frames can be seen as a transformation of qualitative boundaries into the output reference frame that exclude certain values from the interpreta- 


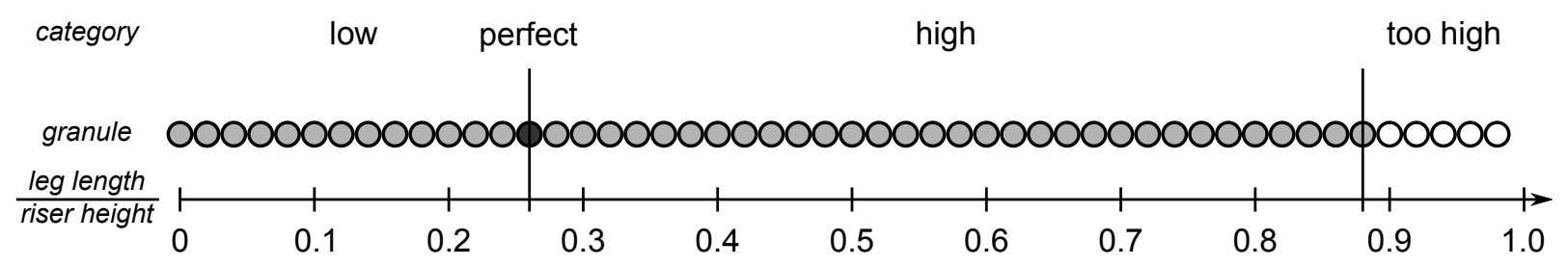

Fig. 4. Reference frame for stair-climbability with granular categories demarcated by the critical and optimal point, atomic granules of perception and metric scale as applied by Warren [46]. The illustration is inspired by [Figure 7.1 in 32, p. 89].

tion of the value in the output reference frame.

$$
f^{\prime}\left(p_{I}, p_{O}, v_{I}\right)=\left\{\begin{array}{l}
\text { "low" } \\
\text { if } p_{I}<p_{O} \text { and } v_{I}=\text { "low" } \\
\text { or "perfect" } \\
\text { "low" or "perfect" or "high" } \\
\text { if } p_{I}<p_{O} \text { and } v_{I}=\text { "high" } \\
\text { "climbable" or "too high" } \\
\text { if } p_{I}<p_{O} \text { and } v_{I}=" \text { too high" } \\
\text { "climbable" or "too high" } \\
\text { if } p_{I}>p_{O} \text { and } v_{I}=\text { "low" } \\
\text { "high" or "too high" } \\
\text { if } p_{I}>p_{O} \text { and } v_{I}=\text { "perfect" } \\
\text { or "high" } \\
\text { "too high" } \\
\text { if } p_{I}>p_{O} \text { and } v_{I}=\text { "too high" }
\end{array}\right.
$$

The function $f$ and $f^{\prime}$ apply to the case that $p_{I} \neq p_{O}$. In the case of $p_{I}=p_{O}$ the transformation function is the identity function. The transformation functions $f$ and $f^{\prime}$ are not uniquely defined as mappings into only one of the original values, but there exist cases where the original values are mapped to the union of two or more values.

The practical benefit of a datum transformation for semantic web applications might seem arguable. In many applications of simple, physical affordances, datum transformations are not necessary because the agents and the objects in the environment are sufficiently modelled to allow direct computations of the ratios that stand for the affordance. For example, in an ambient assisted living apartment all objects that afford climbing, in particular the stairs, are known with their height. Therefore, the climbability affordances for a new resident could be calculated when the new resident's length is known. In many cases, such an apartment is already designed according to the parameters of prospective residents.

However, reference systems for simple, physical affordances can be used as a common ground for da- tum parameters of more complex affordances. Furthermore, artificial agents, such as robots, which are usually equipped with technical sensors, can benefit from datum transformations. ${ }^{13}$

\subsection{Direct Transformation}

A direct transformation requires the identification of values denoting the same entity in the world in different reference frames. The identification of this entity assumes the existence of a reference system for the entity that is shared across the agents who define the input and output reference systems. Given a set of entities whose values for the observed affordance are known in the original and in the target reference frame, there are different ways to derive the transformation function. This work uses a method of transformation that is closely aligned with the method of coordinate transformation in Surveying [cf. 18,13]. In the following the representation of values, the establishment of the transformation function and a small example are discussed.

\subsubsection{Representing the Values for Direct Transformations}

To allow for a direct transformation the representations of the values have to be brought into an interoperable form of representation. As there are no constraints on this in the egocentric reference frame, it cannot be assumed that the values and terms in one reference frame are convertible into the values and terms of another reference frame in their respective form. This article employs a simple vector form, as used in Information Retrieval [37], to represent the values of the ordinal reference frame. The vector's dimension is equal to the number of possible values. Such a vector allows to better deal with the discrete nature of the values than a single value for the rating. The vector's dimensions are

\footnotetext{
${ }^{13}$ see for instance $[36,35]$ for an example of a system of agents interacting with each other and with the environment and [34] for a functional affordance-based model of a robot.
} 
linearly independent. The linear independence of the vectors dimensions causes a loss of information about relations between the values. In particular, the ordering among values is not reflected. An extended model for vector representation that allows linear dependence of values has been suggested in [47]. To avoid overly increasing complexity of our transformations this model has not been explored for this work.

As the vector representation is rather space consuming on paper, a simpler example than the six-value reference frame of hiking paths is used. ${ }^{14}$ The reference frame has three values T1 ("Valley Hike"), T2 ("Mountain Hike") and T3 ("Challenging Mountain Hike"). These values correspond to the canonical unit vectors as follows:

$$
\mathrm{T} 1=\left[\begin{array}{l}
1 \\
0 \\
0
\end{array}\right], \quad \mathrm{T} 2=\left[\begin{array}{l}
0 \\
1 \\
0
\end{array}\right], \quad \mathrm{T} 3=\left[\begin{array}{l}
0 \\
0 \\
1
\end{array}\right]
$$

It has to be kept in mind that even though vectors with numbers are used, the values are not given on a metric scale, but they still represent ordinal values. For computations, the simplest way is to interpret the zeros and ones in the vector as boolean values, so that the canonical unit vectors would represent only one value. However, transformations can result in vectors different from those depicted in Equation 3. A vector resulting from a mapping from $\mathrm{T} 1_{I}$ in the input reference system to either $\mathrm{T} 1_{O}$ or $\mathrm{T} 2_{O}$ would have a 1 in the dimension for $\mathrm{T} 1$ and $\mathrm{T} 2$. These examples would be interpreted into the following predicates:

$$
\left[\begin{array}{l}
1 \\
0 \\
0
\end{array}\right] \rightarrow \mathrm{T} 1 \wedge \neg \mathrm{T} 2 \wedge \neg \mathrm{T} 3, \quad\left[\begin{array}{l}
1 \\
1 \\
0
\end{array}\right] \rightarrow \mathrm{T} 1 \vee \mathrm{T} 2 \wedge \neg \mathrm{T} 3
$$

A slightly more expressive interpretation of the vectors in Equation 3 takes the component values as probabilities of value membership. The vectors in Equation 3 express that the rating is $100 \%$ certain. This is reasonable for the user-ratings, which are directly contributed by the user. The move to interpret the numbers in the vector not as the ordinal value but as probability for an observable is a mathematical "trick" [2, p. 106]. It allows for quantitative computations on the values that are not possible with the original ordinal values. However, this has to be kept in mind through-

\footnotetext{
${ }^{14}$ The reference frame used in the implementation has six values. Only the first three values are used here.
}

out the transformation. In the initial case when users give a rating according to a predefined scheme of ordinal or nominal values, the values correspond to the canonical unit vectors given in Equation 3. The resulting vectors can carry probability values that must add up to $1 .{ }^{15}$ For example, it is possible to express that the transformed rating of the path is $\mathrm{T} 1$ with a probability of 0.8 and T2 with a probability of 0.2 in a target reference frame:

$$
\left[\begin{array}{c}
0.8 \\
0.2 \\
0
\end{array}\right] \rightarrow(\mathrm{T} 1,0.8) \wedge(\mathrm{T} 2,0.2) \wedge(\mathrm{T} 3,0)
$$

The vector represents a fuzzy predicate, and a translation back into a reference frame with non-fuzzy values is only possible by giving up the information about the probabilities or by approximating it through a fixed set of special predicates. Alternatively, the reference frame can be modelled to take fuzzy values.

\subsubsection{Establishing the Transformation Function}

Given the algebraic representation of the values as vectors, the transformation function takes the form of a weight matrix. The weight matrix is multiplied with the input vectors and results in the output vector, which represents the value in the target reference frame. Again, the mathematical approach is closely related to well-known approaches for coordinate transformations in Surveying [e.g. 18,13]. The general formula is written down as follows:

$$
O=W * I
$$

Writing out the vectors and the weight matrix the general form of the equation looks like this:

$$
\left[\begin{array}{c}
O_{1} \\
\vdots \\
O_{m}
\end{array}\right]=\left[\begin{array}{ccc}
w_{11} & \cdots & w_{1 n} \\
\vdots & \ddots & \vdots \\
w_{m 1} & \cdots & w_{m n}
\end{array}\right] *\left[\begin{array}{c}
I_{1} \\
\vdots \\
I_{n}
\end{array}\right]
$$

The matrix $W$ is an interaction matrix [2] between the two sets of values given by the two users for the shared observables. It contains weights that state which impact the various components from the input vector have on the output vector. $w_{i j}$ models the impact that

\footnotetext{
${ }^{15}$ This claim ignores cases where rounding leads to a sum different than 1, for example, when the three values denote one third, which is represented as 0.33 , then the sum only adds up to 0.99 . This problem does not change the theory.
} 
the $\mathrm{j}^{\text {th }}$ component of $I$ has on the $\mathrm{i}^{\text {th }}$ component of $O$. In the general case the matrix does not need to be a square matrix, but can also transform between reference systems with different numbers of values. The major challenge is to find the values for the weight matrix $W$.

In geographic reference systems the matrix is built up by first identifying points whose coordinates are known in both systems. When a sufficient number of points has been identified in both systems, a system of equations is set up and solved to derive the matrix weights.

There is no sufficient number of shared observables for a transformation between semantic reference frames for human observations. A higher number usually results in more accurate results, but there are exceptions to this rule, for example, when the observables are not exactly equivalent. This can be caused by time difference between the observations, a restaurant might be renovated or change the chef, the area of a hotel might change, or a hiking path can be in a different condition in fall than it is in summer. Additionally, in many cases the very high values might not be used for ratings by a user. In the hikability rating scenario the tours given the highest value typically require substantial experience and mountaineering skills, which only few users have. Hence, a matrix with missing weights in some places is the normal case rather than the exception. Additionally, the ratings are not unique as coordinates for locations are. Different observations of affordances are very likely to take the same descriptive value. For a boolean weight matrix this work suggests three simple rules to fill the weight matrix:

(1) $\quad w_{i j}=1$ if there exists a shared referent that is rated with the $j^{t h}$ value in the input reference frame, and that is rated with $i^{\text {th }}$ value in the output reference frame.

$$
w_{i j}=0 \quad \text { if there exist one or more shared }
$$
referents that are rated with the $j^{\text {th }}$ value in the input reference frame, and none of them are rated with $i^{t h}$ value in the output reference frame.

(3) $w_{i j}=\mathrm{NA} \quad$ if there is no shared referent that is rated with the $j^{t h}$ value in the input reference frame the weight is assigned the value "NA" for not available.

An approach to reduce the number of unspecified places is to exclude the dimensions that represent val- ues that the user does not use. The changes in the components have to be considered when establishing the matrix.

Moving to a weight matrix for the transformation of vectors that represent probabilities, rules (2) and (3) remain the same as compared to establishing a boolean weight matrix, only rule (1) has to be adapted:

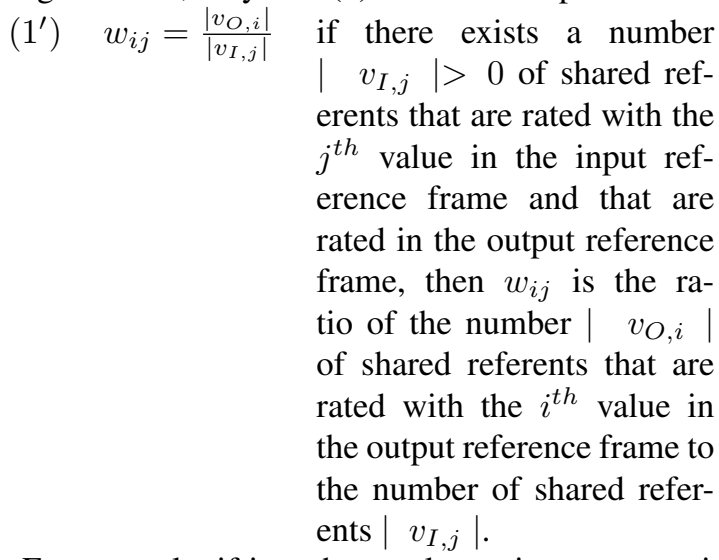

For example, if in a three value rating system with values $\mathrm{T} 1, \mathrm{~T} 2, \mathrm{~T} 3$ there are three tours rated $\mathrm{T} 1_{I}$ in the input reference frame, and these tours are rated $\mathrm{T} 1_{O}$, $\mathrm{T} 1_{O}$ and $\mathrm{T} 2_{O}$, then $w_{11}=0.67$ and $w_{21}=0.33$, rounded to the second decimal place.

\subsubsection{Example}

In the following example, we consider two avid hikers -Ian and Otis- who both completed six similar paths and rated their individual experiences. Otis would like to hike a seventh path that Ian has hiked before and needs a transformation of Ian's rating into his own rating. Table 1 shows the values Ian and Otis assigned to the hikabilities of the paths. The table also shows that the entity in the real world that Ian refers to as Path 1 must be the same as the entity that Otis refers to as Path 1. This assumes an underlying allocentric reference frame for the paths (not their affordances) as reference objects.

Table 1

Values resulting from Ian's and Otis' tour ratings.

\begin{tabular}{lll}
\hline & $\mathrm{Ian}$ & Otis \\
\hline Path 1 & $\mathrm{T} 1_{I}$ & $\mathrm{~T} 2_{O}$ \\
Path 2 & $\mathrm{T} 1_{I}$ & $\mathrm{~T} 1_{O}$ \\
Path 3 & $\mathrm{T} 2_{I}$ & $\mathrm{~T} 3_{O}$ \\
Path 4 & $\mathrm{T} 2_{I}$ & $\mathrm{~T} 2_{O}$ \\
Path 5 & $\mathrm{T} 1_{I}$ & $\mathrm{~T} 1_{O}$ \\
Path 6 & $\mathrm{T} 3_{I}$ & $\mathrm{~T} 3_{O}$ \\
Path 7 & $\mathrm{T} 2_{I}$ & \\
\hline
\end{tabular}


To fill the weight matrix, the probabilities of a mapping from $\mathrm{T}_{I}$ to values in $O$ are calculated from the given matches. In the example the three paths rated with the value $\mathrm{T} 1_{I}$, are rated two times with $\mathrm{T} 1_{O}$ and once with $\mathrm{T} 2{ }_{O}$. According to rule (1'), the weight $w_{11}$ is constructed as follows: There exists a path rated with value 1 (T1) in the input reference frame (Path 1, 2 and 5) and two of these paths (Path 2 and 5) are rated with value 1 (T1) in the output reference frame, which gives a ration of $2 / 3$ for the weight $w_{11}$. Likewise, there exists one path (Path 1) among the three paths rated $T 1_{I}$ that is rated with value 2 (T2) in the output reference frame. This gives a ratio of $1 / 3$ for the weight $w_{21}$. This leads to a mapping of $\mathrm{T}_{I}$ as follows (the components are rounded to the second decimal place):

$$
\left[\begin{array}{l}
1 \\
0 \\
0
\end{array}\right]_{I} \rightarrow\left[\begin{array}{c}
0.67 \\
0.33 \\
0
\end{array}\right]_{O}
$$

The mapping expresses that a path that is rated with $\mathrm{T} 1_{I}$ is rated with a probability of 0.67 as $\mathrm{T} 1_{O}$ and with a probability of 0.33 as $\mathrm{T} 2{ }_{O}$. Applying rule (1') to each weight in $W$ the resulting weight matrix looks like this:

$$
W=\left[\begin{array}{ccc}
0.67 & 0 & 0 \\
0.33 & 0.5 & 0 \\
0 & 0.5 & 1.0
\end{array}\right]
$$

Hence, Path 7 would result in a probabilistic rating of 0.5 for $\mathrm{T} 2{ }_{O}$ and 0.5 for $\mathrm{T} 3_{O}$ :

$$
\left[\begin{array}{c}
0 \\
0.5 \\
0.5
\end{array}\right]_{O}=\left[\begin{array}{ccc}
0.67 & 0 & 0 \\
0.33 & 0.5 & 0 \\
0 & 0.5 & 1.0
\end{array}\right] *\left[\begin{array}{l}
0 \\
1 \\
0
\end{array}\right]_{I}
$$

As a result, a theory that allows to account for the values of individual user-ratings taken with reference to the user's specific capabilities is established. Furthermore, the transformations allow to estimate additional ratings, based on transformations from a second user's values into the first user's reference frame.

\section{Egocentric Semantic Reference Systems for Semantic Web Applications}

In the introduction it was hypothesized that an egocentric semantic reference system for human observations of affordances allows capturing the subjec- tive meaning of observation values in ordinal reference frames. This section presents a prototypical implementation of an egocentric semantic reference system. The prototype illustrates at hand of a social web application how user ratings can be represented accurately and ontologically correct, how this helps to overcome the challenges of individual and subjective ratings and how these ratings can be transformed across the users' ordinal reference frames without making unnecessary and invalid assumptions about the user's reference frame and its values.

Throughout this section typewriter font will be used to indicate $\mathrm{RDF}^{16}$ nodes like User. RDF classes start with a capital letter, RDF properties start with a lower case letter.

\subsection{The hikr.org Dataset}

After crawling the data from the hikr.org website, the proprietary stored data have been processed according to the Linked Data principles [1] and stored as an RDF-graph. The Linked Data approach focuses on linking data items and datasets and describing them by reference to URIs. It allows representing the hiking dataset in a flexible way, which also complies to semantic web standards. The adherence to semantic web standards such as RDF and SPARQL ${ }^{17}$ also increases the reproducibility of the results. The original data is retrievable through the hikr.org portal.

The graph structure of the dataset is depicted in Figure $5{ }^{18}$ The respective properties and classes have been added in the processing step, aiming to approximate the structure of the dataset as presented on the website as closely as possible. The total number of triples added up to 552912 and is stored in a Jena ${ }^{19}$ triple store with a fuseki SPARQL server. The SPARQL endpoint ${ }^{20}$ allows to query the RDF graph through the SPARQL Query Language for RDF. The RDF graph has five classes: Tour, Waypoint, User, Region and HikingDifficulty. The class Region is not used in this work. Additionally, the individuals of these classes are connected through relations, i.e. RDF object properties. Each

\footnotetext{
${ }^{16}$ The Resource Description Framework (RDF), see http:// www. w3. org/RDF/

${ }^{17}$ see http: / / www.w3 . org/TR/rdf-sparql-query/

${ }^{18}$ The concept maps have been created in CMap Tools, available for download at http: / / cmap.ihmc.us /

${ }^{19}$ See http: / / jena. apache.org/index.html

${ }^{20}$ At http://spatial.linkedscience.org/sparql
} 


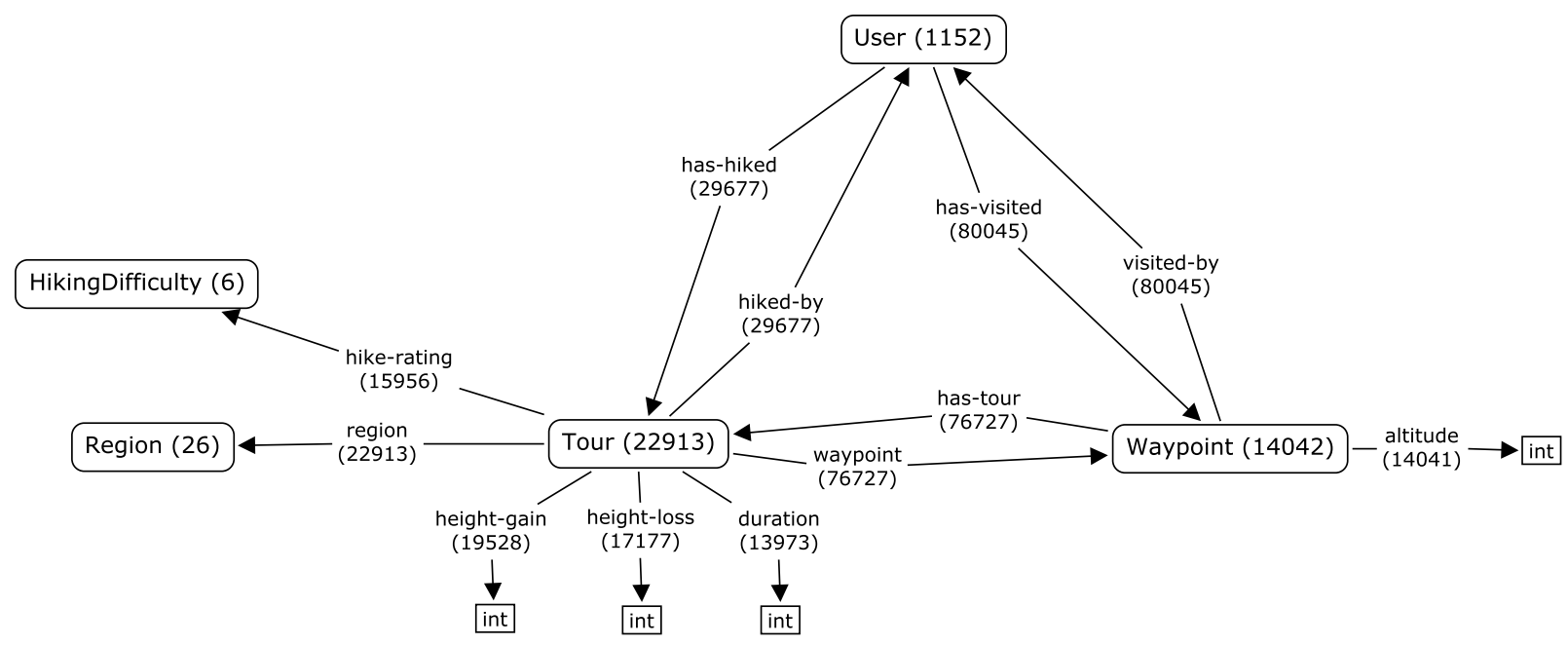

Fig. 5. Concept map illustrating the RDF dataset of hiking tours. The numbers in brackets present the number of individuals for classes and the number of subject-property-object triples for properties. Large round boxes represent classes, small square boxes represent datatypes.

Tour individual, which represents a rated tour is related through hike-rating to an individual of HikingDifficulty. User individuals are related to Tour individuals through the relation has-hiked, and the inverse relation hiked-by. Similar relations has-visited and visited-by exist between User individuals and Waypoint individuals. Waypoint individuals are assigned to Tour individuals through the waypoint relation, and through the inverse has-tour relation. The datatype properties height-gain, height-loss and duration are specified for the individuals of the class Tour. The Waypoints can have a datatype property a l $t$ i $u d e$. Additionally, all individuals have a datatype property title from the Dublin Core Metadata Element $\operatorname{Set}^{21}$.

\subsection{Establishing an Egocentric Semantic Reference Frame and Datum}

To establish a semantic reference system for observations of affordances, this work draws from previous work by Probst [32,33] and is loosely aligned to DOLCE. DOLCE models observable qualities in a socalled EQQS-pattern [25], wherein a quality (Q) inheres in an entity (E). The magnitude of the quality is a quale $(\mathrm{Q})$, which is a region in a quality space $(\mathrm{S})$. Probst [32,33] suggests modelling qualities according to the DOLCE pattern and then built a reference frame as a second layer of representation of qualities and qualia in a partitioned reference space. This model cor-

\footnotetext{
${ }^{21}$ see http: //dublincore.org/documents/dces/
}

responds closely to Bunge's [2] analysis of measurement, which distinguishes properties (DOLCE qualities) from property degrees (DOLCE qualia), which are mapped to magnitude units (Probst's reference regions), which then are ordered on a scale (Probst's reference space). The ontology design pattern for referential qualities [27] together with Probst's [32,33] reference spaces allows to establish an egocentric semantic reference frame and a semantic datum for affordances.

The prototypical implementation of the egocentric semantic reference system makes one important simplification. The implementation does not make the distinction between qualia and quality spaces on the one hand and reference regions and reference spaces on the other, which is suggested by Probst $[32,33]$. Instead, the implementation only requires a part of the DOLCE pattern and models the qualia as values and the quality spaces as reference frame. This simplification comes with a significant reduction in the complexity and size of the implementation. This simplification does not affect any of the reference system's parts.

The datum in the egocentric reference system is given through the observer. The ontology design pattern for referential qualities [27] allows to include the observing agent as a required entity for the observed affordance. Ergo, the observer as datum is accounted for. In the case of the hikability example, no datum parameters, i.e. qualities of the observer, are specified. However, the explicit consideration of the observing agent is manifested in modelling every individual observer with its own egocentric semantic reference frame. That is, instead of six values for the whole 
dataset, there are six values for each user in the implementation of the egocentric semantic reference systems for hikability affordances.

Classes and individuals that represent the affordancequality, the quale and the quality space have to be added. The extended EQQS pattern requires at first an entity in which the quality inheres, i.e. the thing that provides the affordance. The implementation uses the class that originally was intended to represent the tour report.

Here, one could argue that the assignment of a difficulty to a tour report is ontologically inaccurate because the path is rated and not the tour report. For the hikability affordance a new class Hikability is created along with individuals for every rating given by a user. As the user is an integral part of the hikability affordance, paths hiked by more than one user, also have more than one hikability affordance. This leads to 29677 individuals for the hikability affordance, each related to a path and a user.

The classes so far represent the affordance as a quality but not yet the rating. The actual rating that is assigned to the affordance is the quale (the class hQuale). Since not all paths were rated in the original dataset, only 20559 out of 29677 Hikability individuals have a quale assigned to them. A quality has one quale in each reference frame in which it is observed. Given 1152 users, there are 1152 egocentric frames with six values each. Therefore, there exist 6912 hquale individuals, which are part of the 1152 respective quality spaces (class hQSpace).

The dataset modelled with reference frame and datum is depicted in Figure 6. In total, the tripleset including the semantic reference frame is with 715241 triples about $30 \%$ bigger than the original dataset. The reference frame and the datum capture the semantics of human judgements of the hikability affordance that a mountain path affords to a hiker. The human judgements are given as terms referring to six possible values. The meaning of the term is grounded in the user, and by extension in the user's use of these terms to refer to specific paths in the mountains. The resulting category of individual judgements with the same value establishes the extension of the term's meaning for a user.

\subsection{Performing Semantic Transformations}

The final step to an operational notion of affordance observation is the implementation of semantic transformations. Unfortunately, there is far less previous work that can be drawn from. Therefore, some pragmatic simplifications have to be made throughout the establishment of semantic transformations.

There are 1152 reference frames in the dataset, and transformations are specific to each pair of two reference frames. Not only the sheer amount of possible transformation functions, but also the dynamic nature of community driven portals, with an ever changing (increasing) dataset impedes a pre-computation of weight matrices. To provide an idea of a typical application that requires a semantic transformation a small scenario is chosen:

Rita plans to visit a friend in Lucerne and would like to hike up the famous Rigi Mountain close by. As she has never hiked in this region before, she would like to know how difficult it would be for her to hike the Rigi (and maybe even which route to take).

The waypoint for Rigi Kulm ${ }^{22}$, the top of the Rigi Mountain appears in 91 tour reports. 89 of these reports are rated, with ratings ranging from value $\mathrm{T} 1$ to T5. Rita is a frequent user of the hiking portal, she contributed 92 reports to the portal. 85 of her reports are rated according to the given six-value rating scale. Rita has hiked mostly in the Canton of Ticino.

The matching of tours poses an additional challenge in the hikr dataset. Each tour report is unique, and refers to a specific hike on a hiking path at a certain date. A shared reference frame for hiking path referents in the real world is missing. Therefore, the approach here has to resort to the waypoints assigned to the hiking path. The waypoints are predefined in the hiking portal, and the set of waypoints can be considered an allocentric reference frame. The matching of paths can only be performed at hand of a similarity measure that allows to treat sufficiently similar paths as equivalent. This work uses an overlap of waypoints among paths as naive measure for the similarity of paths.

To derive an expected rating of a path to the Rigi in Rita's reference frame the following steps have to be performed:

1. Find users that have hiked paths that include the waypoint for Rigi Kulm.

2. Find the waypoints that these users have hiked.

\footnotetext{
${ }^{22}$ Waypoint Rigi Kulm: http://www.hikr.org/dir/ Rigi_Kulm_4131/
} 


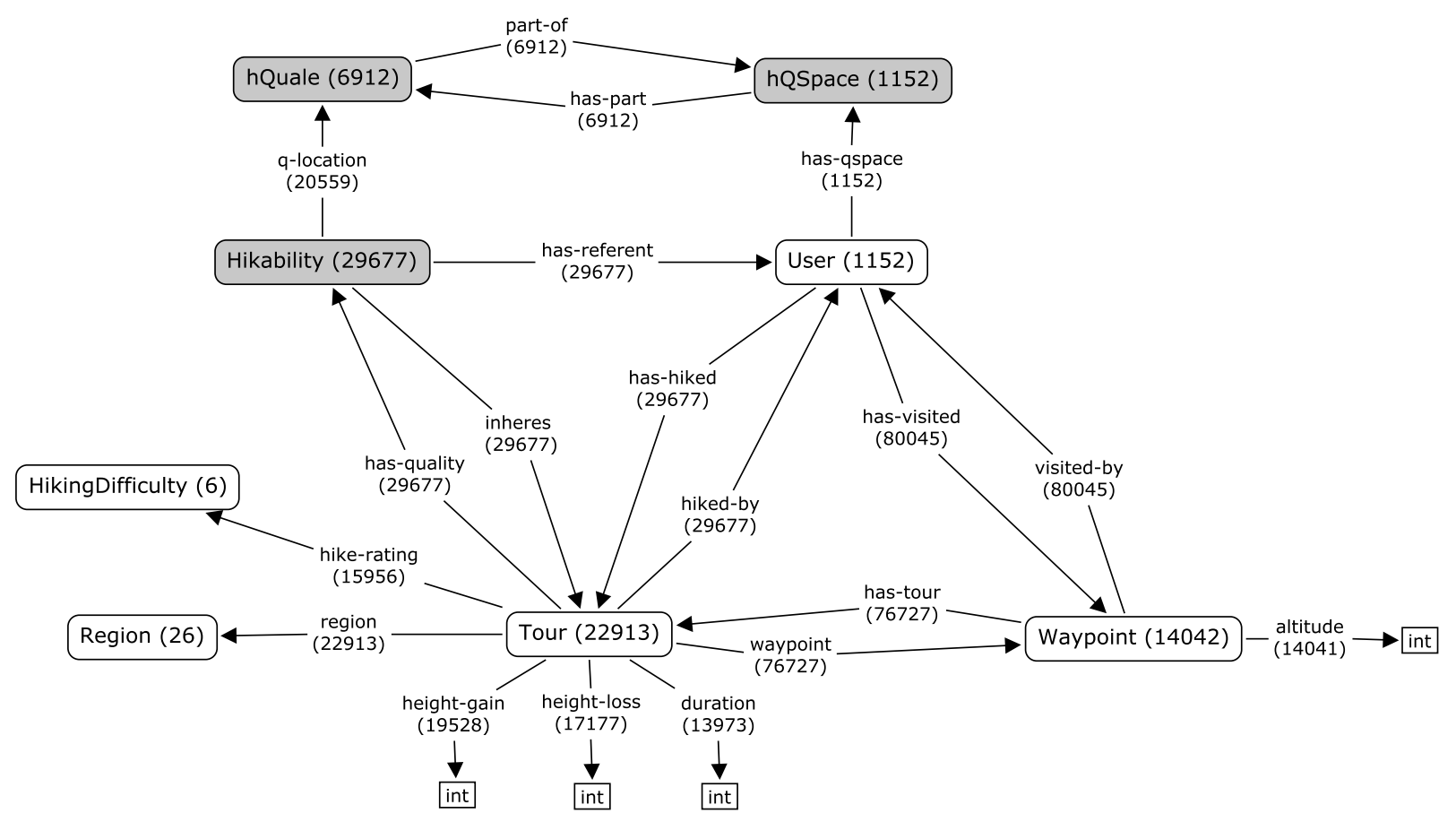

Fig. 6. Concept map illustrating the RDF dataset of hiking tours including the reference frame for hikability. Classes of the original hikr.org dataset are depicted in white, the classes for the reference frame extension are shown in grey. Properties between individuals are depicted as arrows between the classes of individuals.

3. Find matches between Rita's waypoints and the waypoints found in Step 2.

4. Rank the users resulting from Step 1. according to the number of matches found in Step 3.

5. Select the highest ranked user from Step 4. and fill the matrix according to the three rules stated above (cf. Section 5).

The first two steps can be solved with SPARQL queries to the triplestore. The respective queries are shown in Listing 8 and Listing 8 in the Appendix. Steps 3 and 4 are more complex and are explained in the following.

\subsubsection{Similarity-based Tour-matching}

The ratings in the hiking portal face one problem that other rating portals (for example, for hotels or restaurants) do not have: Users do not rate the same observable over and over again, but users rate their own individual hiking tour. For the semantic translations, we have to make the assumption that the user's rating of the tour is actually a rating of the hiking path or route that this tour comprised. Thereby, the rating abstracts from temporal influences, such as varying conditions due to different seasons. Yet, even with this assumption equivalent paths have to be identified among the individual reports. In the hikr.org portal a set of waypoints is predefined, so that users can assign the same waypoints to their individual tour report. The waypoints that different paths have in common can be exploited to come up with a simple similarity measure. Sufficiently similar paths are potential candidates of shared observables for the weight matrix. A simple algorithm matched the paths according to their common waypoints. However, a closer examination also revealed that the user Rita, has hiked the same paths several times. To preclude a bias towards certain paths, only the most recent tour of a set tours with equivalent paths is taken into account.

The similarity based strategy bears some peculiarities. In a heterogeneous landscape such as mountains, an additional waypoint, maybe an additional peak, can add substantially to the difficulty. There might also exist several paths of different difficulties between the same two waypoints. Moreover, the paths have very different total amounts of waypoints, ranging from zero to more than ten. First queries indicated that the paths seldom share more than four waypoints. To obtain a sufficient number of equivalent paths the threshold for assumed equivalence of paths was set to two shared waypoints. This is very low and comes with a 
large degree of uncertainty, but for the sake of the prototype and as proof of concept this uncertainty is accepted.

The peculiarity is mostly induced by the automatic matching that even exceeds the state of the art for geographic reference systems. Even today, direct transformations are usually based on shared points that have to be identified manually. A manual identification, or at least verification, is possible, but requires more user interaction and was not feasible for the example in this work.

All users that have hiked a path, which includes the waypoint Rigi Kulm are querried. The result is a set $U$ of 65 users. For each user $u_{i}$ in $U$, the waypoints that this user has visited are queried returning sets $W_{i}$ with waypoints. Each set of waypoints $W_{i}$ is compared to Rita's set of waypoints $W_{\text {Rita }}$ and the users $u_{i}$ are ranked according to the number of waypoints they have in common with Rita. The highest ranked user is selected as suitable candidate to establish the transformation matrix between this user's reference frame and Rita's reference frame. In our implementation the highest ranked user was named "chaeppi”. He has 56 waypoints and 13 paths in common with Rita.

\subsubsection{Establishing the Matrix}

Given user chaeppi and his paths that match one of Rita's paths, the weight matrix can be established. First the ratings for the paths are queried for Rita and for chaeppi to derive rating pairs, which reflect the unique paths rated in Rita's and in chaeppi's reference frame. From these pairs the probabilities for the weight matrix can be calculated according to the rules (1'), (2) and (3) specified in Section 5. The resulting matrix is shown in Equation 11, again rounded to two decimal places.

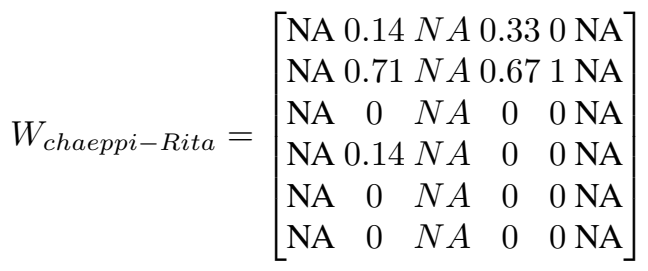

The matrix is incomplete, because chaeppi's did not use the values T1, T3 and T6 in any of the shared paths. The weights that cannot be specified are marked NA in the matrix.

The final step is to query chaeppi's path, which included the waypoint Rigi Kulm and its rating, and to project this rating into Rita's reference frame. User chaeppi has rated the path he took to the Rigi Kulm waypoint with T2. The transformation into Rita's reference frame is shown in Equation 12.

$$
\left[\begin{array}{c}
0.14 \\
0.71 \\
0 \\
0.14 \\
0 \\
0
\end{array}\right]=\left[\begin{array}{ccccc}
\mathrm{NA} & 0.14 & N A & 0.33 & 0 \mathrm{NA} \\
\mathrm{NA} & 0.71 & N A & 0.67 & 1 \mathrm{NA} \\
\mathrm{NA} & 0 & N A & 0 & 0 \mathrm{NA} \\
\mathrm{NA} & 0.14 & N A & 0 & 0 \mathrm{NA} \\
\mathrm{NA} & 0 & N A & 0 & 0 \mathrm{NA} \\
\mathrm{NA} & 0 & N A & 0 & 0 \mathrm{NA}
\end{array}\right] *\left[\begin{array}{l}
0 \\
1 \\
0 \\
0 \\
0 \\
0
\end{array}\right]
$$

Hence, in our scenario the path that chaeppi took up to the Rigi mountain, would be rated with $71 \%$ probability as T2 by Rita, and only with $14 \%$ probability as T1 and with another $14 \%$ probability as T4 (1\% percent has been lost to rounding errors). The implementation of the example in $\mathrm{R}$ is documented online $\mathrm{e}^{23}$.

\subsection{Comparison of transformed ratings to actual ratings}

The example has shown that the method works and results in a vector of probabilities. The probabilities indicate how likely the user would perceive the suggested path in the categories of her egocentric reference frame. To gain an insight if the method also yields results that actually match the users actual perception, we have conducted a few more tests in our dataset.

After comparing the matches of tours across all users we have selected four pairs of users that have twenty or more matching tours and that have not hiked too many tours together, using an equivalence measure of four shared waypoints among the tours. Table 2 shows the pairs of users with the number of matching tours and the overlap between the users. The overlap is the number of tours that were hiked together by the two users and that are reported and rated in the same tour report.

Table 2

User pairs with matching tours used for the comparison of transformed and actual ratings and the number of tours that were hiked together by the two users.

\begin{tabular}{llll}
\hline User 1 & User 2 & Matches & Overlap \\
\hline Lena & chaeppi & 20 & 0 \\
Mauro78 & beppe & 22 & 0 \\
Bombo & rgauss & 25 & 8 \\
Ivo66 & alpstein & 66 & 9 \\
\hline
\end{tabular}

For each pair of users we have calculated the matrices for each subset of matching tours that lacks one

${ }^{23}$ see http: //www. jensortmann. de/R/ers.html 
tour. For example in the case of 20 matching tours, we have computed 20 transformation matrices, each based on a subset of 19 tours. We have then calculated the transformed rating of the second user by multiplying the vector representation of the first users's rating with the transformation matrix. We have then compared the transformed rating with the actual rating of the user. Table 3 shows the accuracy of transformation results.

Table 3

Accuracy of the transformed rating between User 1 and User 2. The column "+/- 0" indicates the ratio of correct projections. The column "+/- 1" lists the ratio of transformations that were off by at maximum one category in the ordinal rating scale.

\begin{tabular}{llll}
\hline User 1 & User 2 & $+/-0$ & $+/-1$ \\
\hline Lena & chaeppi & 0.525 & 0.725 \\
Mauro78 & beppe & 0.492 & 0.929 \\
Bombo & rgauss & 0.636 & 0.856 \\
Ivo66 & alpstein & 0.447 & 0.869 \\
\hline
\end{tabular}

For example, the projected rating from Lena's reference frame into chaeppi's reference frame resulted in $52.5 \%$ of the cases in the actual rating that chaeppi gave to the tour. In $72.5 \%$ of the cases the rating resulted in the actual category or in an adjacent category. The best accuracy for matches +/- 0 among the four test cases was achieved between the users Bombo and rgauss. $63.6 \%$ of the projected ratings matched the actual rating. However, the two users had hiked 8 of the 25 tours used to compute the matrices together. Among the four tested pairs of users, the highest percentage for projected ratings that are only one rating off was found between Mauro78 and beppe. $92.9 \%$ of the projected ratings were in the actual or in the directly neighbouring category and only $7.1 \%$ of the projected ratings were off by more than one category.

The results show that the projected ratings are plausible and that we can capture the subjective interpretation of the users' hikability ratings. Thereby, the results also validate the theory of egocentric semantic reference systems. Given the simplifying assumptions we have made, there is space for improvements that yield more accurate results in real applications.

\section{Discussion}

In this section the theory of egocentric semantic reference systems is discussed. First, we closely examine the theory. We show what has been achieved and what is possible with the theory that was not before, but also point to remaining shortcomings and persisting problems. Then, the implementation of the theory for the affordances of hikability is discussed. After that, we point to some caveats and offer a critique of the work done.

\subsection{Discussion of the Theory}

The theory of egocentric semantic reference systems for affordances provides a solution to describe, interpret, compare and integrate the results of human observations of affordances. The suggested theory is the first attempt to capture the ontologically challenging notion of affordances in a reference system. The presented theory is compatible with existing theories for affordances [e.g. 14,15,42,46,45], theories of human judgements in information systems [e.g. $48,7,9,8,49,50,51]$ and theories of reference systems [e.g. 2,18,22,23,32,33,13,19]. Important tenets, for example, 'affordances are subjective' [15]; 'the environment is shared' [15] and 'the observer serves as referent of its observation' [15] are found compatible with the suggested theory of egocentric reference frames for affordances. In addition to that, our work corroborates the findings in [39] that observations can be grounded in observation procedures. Furthermore, the suggested egocentric semantic reference frames can be seen as knowledge patterns in the sense of [12], which capture task-specific knowledge and at the same time facilitate ontology design.

The reference system for affordances is developed from an information science and semantic web perspective. The focus is on the symbolization of the observed affordances and not on attending to them in the form of performing actions. Thereby, the actionrelatedness of an affordance fades into the background. Instead the expression and communication of an affordances gains centerstage. The presented theory allows to semantically integrate human observations of affordances across different observers. Furthermore, the compliance with previous work on semantic reference systems fulfils a major requirement for the integration with other reference systems of observations and measurements. A prototypical implementation has demonstrated the validity of the theory, the practical applicability of the approach and the plausibility of the projected ratings.

Specifically, an egocentric reference system offers the opportunity to account for subjective observations. An explicit consideration of the observer and the subjectivity of his/her observations is not possible with 
current allocentric reference systems. Thereby, the presented theory offers a whole range of new opportunities to deal with human observations as information sources. Akin to Freksa [7,8] and Freksa and López de Mántaras [9], making explicit the peculiarities of human observations ultimately leads to more accurate results and the increased knowledge about the observations provenance leads to more trustworthy information sources. With egocentric reference systems for human observations of affordances it is possible to represent ordinal reference frames of observations and to translate observations across ordinal reference frames. The theory allows to suggest transformed categorical values and probabilities thereof. We avoid assumptions of interval scales for our ordinal reference frames, which are often made in current rating websites where users rate on an ordinal reference frames, but the presented average rating assumes an interval scale. The example of a reference frame for stair-climbability illustrates that this assumption cannot simply be made. Finally, the presented approach to transform across different observations of affordances makes these observations operational. The transformation provides a solution to the challenge of subjectivity, thereby refuting a possible conjecture such as: subjective observations are not comparable. Through transformations, users have access to other users' observations in a novel way, which respects the users' own reference frames and values.

Even though we find that the theoretical contribution of this article achieves our goals, there remain several opportunities for future expansion and improvements. Transformation across reference frames of different observers are only discussed as to achieve the minimal result. Especially, the consideration of the ordering across values during the transformation is a desideratum for more reliable applications. Additionally, the establishment of the transformation function can be more sophisticated, for example by weighting the shared observables.

Furthermore, the recent investigations in map scales [e.g. 4] and resolution of sensor observations [e.g. 6] are worthwhile exploring for improving the semantic integration of egocentric semantic reference system using different scales.

\subsection{Discussion of the Semantic Web Application}

With the prototypical implementation of a semantic reference system we have proven that the theory can be put in practice and that it yields reasonable results. It has been shown that the semantic reference system for human observations of hikability affordances allows to capture the semantics of subjective human observations and that we can perform semantic translations of values from one user's reference frame into another user's reference frame. The transformed results have been found to correspond to that user's rating. Yet, the transformation makes simplifying assumptions and only employs a very simple transformation function. Thus, there is room for improvement that allows to make the projections even more accurate.

One simplification is the reduction to qualia and quality spaces to represent ratings, instead of using the full system of qualia, reference regions, quality spaces and reference spaces suggested by Probst $[32,33]$. Probst, akin to DOLCE, models a quality that inheres in a host and is mapped to a quale in a quality space. Though the quality is individual to the host, several individual qualities can be mapped to the same quale. Qualia are then mapped to reference regions in reference spaces. A reference region is a unit in a reference frame. Through the structure and granularity of reference frames, several qualia map to the same reference region. The distinction of qualia and reference regions is reasonable for allocentric reference frames, where the qualia of different agent's can be mapped to a shared reference frame. However, in the case of an egocentric reference system, the qualia and reference regions are both individual to the agent. Furthermore, the exact mapping of qualia to reference regions in the egocentric reference frame is internal to the agent. There is no need to replicate this mapping. Instead, qualia can be used as reference regions. Reference regions as well as qualia are both subcategories of regions in DOLCE. Thereby, our implementation of an egocentric semantic reference system remains ontologically close enough to allocentric semantic reference frames established as in $[32,33]$. This in turn allows semantically integrating the results of egocentric and allocentric reference frames. In addition to that, we avoid unnecessary cognitive assumptions about the internal world of the agent and at the same time have a valid and sound semantic representation of the original hikability dataset. Thereby, a new knowledge pattern [12] that is used as an ontology design pattern [10,11] for egocentric reference frames emerges.

The implementation uses an RDF triplestore to represent semantic reference frames and datums and it uses R to query the triple store with SPARQL and execute the transformation based on the query results. One could argue that (1) R is not a semantic web tech- 
nology, (2) the transformations performed in R do not use reasoning in the semantic web sense and (3) the results of the calculations in $\mathrm{R}$ are not semantically integrated with the original semantic reference frame. While we agree to the first two points, we disagree with the third one. Firstly, the values that are used in $\mathrm{R}$ are always taken as values in the semantic reference frame. Even though the typing is not checked and enforced in $\mathrm{R}$, we have taken care of the type consistency within the $\mathrm{R}$ transformations. Therefore, the resulting values are values that can be interpreted in the semantic reference frames. Secondly, the R scripts are mostly used to perform SPARQL queries on the triple store and to iteratively perform more SPARQL queries on the triple store, eventually counting the results and performing ratios between values of the same type. Thereby, we have manually bridged across the discontinuity between the semantic technologies and the nonsemantic technologies. The manual effort that is required to specify the transformations reduces the flexibility of using $\mathrm{R}$ for semantic interoperability. Semantic interoperability across reference frames remains restricted to the transformation operation that we have specified. A functional language with a stronger typing might allow to better approximate the semantics of the reference frame and datum to offer a better support for a richer semantic interoperability.

The implementation of the egocentric semantic reference system for the hikability affordance does not exceed a prototype stage. Several simplifications have been made and no effort has been put into increasing the performance. Yet, it shows that it is possible with current technologies to establish a full fledged semantic reference system for human observations of affordances that even allows semantic translations across different reference frames in the same reference system.

\subsection{Critique and Caveats of Egocentric Semantic Reference Systems}

The theory of egocentric semantic reference systems is particularly useful for subjective human observations and where the group of users is very heterogeneous. An example are hotel ratings, where the observations are made by people of very different age and with very different backgrounds, such as students, families and pensioners. Another example are ratings of restaurants and dishes where the subjective taste of for example saltiness or spiciness differs strongly across people.
Ratings of physical affordances, for instance the hikability and the stair-climbing affordances fall into a borderline category. On the one side, most humans are equipped with more or less the same physical capabilities. Stairs are standardized in many countries, so that the stairs we encounter are usually easily climbable for everyone. On the other side, physically more challenging activities and activities that require experience and skill are more subjective because fitness and skills can vary strongly from person to person.

Generally, the designer of a reference system has to make a decision how subjective the ratings are, and whether the influence of subjectivity can impact the ratings to a degree that justifies or requires the additional implementation and data storage effort that egocentric semantic reference systems require. The database of prototypical implementation was about $30 \%$ larger after the semantic reference frames had been established, and deriving the matrices for transformations is computationally much more expensive then calculating a simple or weighted mean.

The accuracy currently achieved with the prototype might not suffice for applications where decision with a high risk are based on the transformed ratings. A mountain tour that exceeds the hikers capabilities clearly puts the hiker in a high risk, but the hiker still would have the chance to just turn around. This might for instance not be the case for rock climbing. However, the accuracy of transformations strongly depends on the assumptions made and it has been highlighted that the assumptions made in the prototype are very simple. More sophisticated assumptions about equivalence of tours are likely to increase the accuracy of transformations.

Finally, transformations across categorical reference frames require a rather high number of matches of observables between the observers. For hiking tours with a reference frame of six categories, we found that about 20 equivalent tours are necessary to come up with a conclusive transformation matrix. To have 20 matches between two users, these users must have contributed a significant number of hiking ratings. However, this also introduces a incentive for users to continue contribute reports to the portal.

In summary, the theory of egocentric semantic reference systems for human observations provides a valuable extension to the semantic reference systems. It provides a solution to deal with and to respect the restrictions of ordinal reference frames, where the extends of the values cannot be assumed to be equal. The 
theory is particularly helpful for very subjective human observations.

\section{Conclusion}

This paper began with a more than 2000 year old postulation of "man as measure" by the Greek philosopher Protagoras. This postulation has been put into the context of modern psychological theories of perception and of current contributions of human observations as information sources in social and semantic web application. Inspired by Protagoras bold claim, the paper introduced a theory of egocentric reference systems to information science, and showed how egocentric reference systems allow to capture subjective interpretations of terms and values in semantic web applications.

We have detailed how to specify the semantic datum that grounds a set of values outside the reference system, how to establish the semantic reference frame that covers the observation values an observer uses to denote her observations of affordances and we have suggested a way to derive transformation functions between different egocentric reference frames. The theory has been found valid and a prototypical implementation has shown the practical benefits but also revealed remaining issues and limitations.

The prototypical implementation of a dataset of ratings of mountain tours is the second contribution. Though only intended as a prototype, it is fully usable. The prototype establishes egocentric reference frames for more than 1000 users and specifies more than 20000 ratings in these reference frames. R scripts allow to transform values from one reference frame into another, yielding a vector of probabilities for the ratings in the target reference frame. We have made the dataset available in a triple store with a SPARQL endpoint. An exemplary R script is available online.

Finally, the results of the theory and the prototype have been discussed and critically reviewed. The result of the discussion allows to accept the hypothesis that an egocentric semantic reference system allows to semantically account for human observations of affordances and we conclude that the theory of egocentric semantic reference systems for observations of affordances makes every user the measure of his or her own observations.

\section{References}

[1] Berners-Lee, T. (2009). Linked data. Design issues, W3C. originally published 27.07.2006, http://www.w3. org/DesignIssues/LinkedData.html.

[2] Bunge, M. (1973). On confusing 'measure' and 'measurement' in the methodology of behavioral science. In Bunge, M., editor, The Methodological Unity of Science, pages 105 - 122. D. Reidel Publishing Company, Dordrecht, The Netherlands.

[3] Carello, C., Grosofsky, A., Reichel, F., Solomon, H., and Turvey, M. (1989). Visually perceiving what is reachable. Ecological Psychology, 1(1):27-54

[4] Carral, D., Scheider, S., Janowicz, K., Vardeman, K., Krisnadhi, A., and Hitzler, P. (2013). An ontology design pattern for cartographic map scale. In Cimiano, P., Corcho, O., Presutti, V., Hollink, L., and Rudolph, S., editors, The Semantic Web: Semantics and Big Data. 10th International Conference, ESWC 2013, Montpellier, France, May 26-30, 2013. Proceedings., volume 7882 of Lecture Notes in Computer Science, pages 76-93, Heidelberg, Germany. Springer.

[5] Chrisman, N. R. (2002). Reference systems for measurement. In Chrisman, N. R., editor, Exploring Geographical Information Systems, pages 15-35. Wiley, Hoboken, NJ, 2nd edition.

[6] Degbelo, A. (2013). Modelling the spatial and temporal resolution of a sensor observation. In Proceedings of GI Forum 2013, Salzburg, Austria. to appear.

[7] Freksa, C. (1980). Communication about visual patterns by means of fuzzy characterizations. In XXIInd Intern. Congress of Psychology, Leipzig, Germany, July 6-12 1980. International Union of Psychological Science.

[8] Freksa, C. (1982). Linguistic description of human judgments in expert systems and in the 'soft' sciences. In Gupta, M. and Sanchez, E., editors, Approximate Reasoning in Decision Analysis, pages 297-305. North-Holland Publishing Company, Amsterdam, The Netherlands.

[9] Freksa, C. and López de Mántaras, R. (1982). An adaptive computer system for linguistic categorization of "soft" observations in expert systems and in the social sciences. In Ballester, A., Cardús, D., and Trillas, E., editors, Proceedings of the 2nd World Conference on Mathematics at the Service of Man, Las Palmas, pages 288-292. Universidad Politécnica de Las Palmas.

[10] Gangemi, A. (2005). Ontology design patterns for semantic web content. In Gil, Y., Motta, E., Benjamins, V., and Musen, M., editors, The Semantic Web-ISWC 2005, volume 3729 of Lecture Notes in Computer Science, pages 262-276, Heidelberg, Germany. Springer.

[11] Gangemi, A. and Presutti, V. (2009). Handbook on Ontologies, chapter Ontology design patterns, pages 221-243. International Handbooks on Information Systems. Springer, Heidelberg, Germany, 2nd edition.

[12] Gangemi, A. and Presutti, V. (2010). Towards a pattern science for the semantic web. Semantic Web, 1(1-2):61-68.

[13] Ghilani, C. (2010). Adjustment computations: spatial data analysis. Wiley, Hoboken, NJ, 5th edition.

[14] Gibson, J. (1977). The Theory of Affordances. In Shaw, R. and Bransford, J., editors, Perceiving, acting, and knowing: Toward an ecological psychology, pages 67-82. Lawrence Erlbaum, Hillsdale, NJ.

[15] Gibson, J. (1979). The ecological approach to visual perception. Lawrence Erlbaum, Hillsdale, NJ. 
[16] Harnad, S. (1990). The symbol grounding problem. Physica D: Nonlinear Phenomena, 42(1-3):335-346.

[17] Heft, H. (1989). Affordances and the body: An intentional analysis of Gibson's ecological approach to visual perception. Journal for the theory of social behaviour, 19(1):1-30.

[18] Iliffe, J. (2000). Datums and Map Projections. Whittles Publishing, Caithness, Scottland, UK.

[19] Janowicz, K. and Scheider, S. (2010). Semantic reference systems. In Warf, B., editor, Encyclopedia of Geography. SAGE Publications, Thousand Oaks, CA.

[20] Jones, K. (2003). What is an affordance? Ecological Psychology, 15(2):107-114.

[21] Klatzky, R. (1998). Allocentric and egocentric spatial representations: Definitions, distinctions, and interconnections. In Freksa, C., Habel, C., and Wender, K., editors, Spatial cognition - An interdisciplinary approach to representation and processing of spatial knowledge, volume 1404 of Lecture Notes in Artificial Intelligence, pages 1-17, Heidelberg, Germany. Springer.

[22] Kuhn, W. (2003). Semantic reference systems. International Journal of Geographical Information Science, 17(5):405-409.

[23] Kuhn, W. and Raubal, M. (2003). Implementing Semantic Reference Systems. In Gould, M., Laurini, R., and Coulondre, S., editors, Proceedings of the AGILE 2003 - 6th AGILE Conference on Geographic Information Science, Lyon, France, Collection des sciences appliqueés de l'INSA de Lyon, pages 63-72, Lausanne, Switzerland. Presses polytechniques et universitaires romandes.

[24] Mark, L., Balliett, J., Craver, K., Douglas, S., and Fox, T. (1990). What an actor must do in order to perceive the affordance for sitting. Ecological Psychology, 2(4):325-366.

[25] Masolo, C. and Borgo, S. (2005). Qualities in formal ontology. In Hitzler, P., Lutz, C., and Stumme, G., editors, Proceedings of the KI2005 Workshop on Foundational Aspects of Ontologies, FOnt'05, Koblenz, Germany, September 2005, number 9/2005 in Fachbericht Informatik, pages 2-16. Universität Koblenz-Landau.

[26] Masolo, C., Borgo, S., Gangemi, A., Guarino, N., and Oltramari, A. (2003). Wonderweb deliverable D18 ontology library (final). Deliverable, Laboratory for Applied Ontology - ISTCCNR.

[27] Ortmann, J. and Daniel, D. (2011). An ontology design pattern for referential qualities. In Aroyo, L., Welty, C., Alani, H., Taylor, J., Bernstein, A., Kagal, L., Noy, N., and Blomqvist, E., editors, The Semantic Web - ISWC 2011, volume 7031 of Lecture Notes in Computer Science, pages 537-552, Heidelberg, Germany. Springer.

[28] Ortmann, J. and Kuhn, W. (2010). Affordances as Qualities. In Galton, A. and Mizoguchi, R., editor, Formal Ontology in Information Systems Proceedings of the Sixth International Conference (FOIS 2010), volume 209 of Frontiers in Artificial Intelligence and Applications, pages 117-130, Amsterdam, The Netherlands. IOS Press.

[29] Oudejans, R., Michaels, C., van Dort, B., and Frissen, E. (1996). To cross or not to cross: The effect of locomotion on street-crossing behavior. Ecological Psychology, 8(3):259-267.

[30] Plato (360 BC). Thaetetus. Online translation at http:// www. perseus.tufts. edu/.

[31] Popper (1962). Conjectures and Refutations - The Growth of Scientific Knowledge, chapter Science: Conjectures and Refutations, pages 33-65. Harper Torchbooks. Harper \& Row, New York, NY.
[32] Probst, F. (2007). Semantic Reference System for Observations and Measurements. PhD Thesis, University of Münster, Germany.

[33] Probst, F. (2008). Observations, measurements and semantic reference spaces. Applied Ontology, 3(1-2):63-89.

[34] Raubal, M. and Moratz, R. (2008). A functional model for affordance-based agents. In Rome, E., Hertzberg, J., and Dorffner, G., editors, Towards Affordance-Based Robot Control - International Seminar, Dagstuhl Castle, Germany, June 5-9, 2006. Revised Papers, volume 4760 of Lecture Notes in Computer Science, pages 91-105, Heidelberg, Germany. Springer.

[35] Saffiotti, A. and Broxvall, M. (2008). Affordances in an Ecology of Physically Embedded Intelligent Systems. In Rome, E., Hertzberg, J., and Dorffner, G., editors, Towards AffordanceBased Robot Control - International Seminar, Dagstuhl Castle, Germany, June 5-9, 2006. Revised Papers, volume 4760 of Lecture Notes in Computer Science, pages 106-121, Heidelberg, Germany. Springer.

[36] Saffiotti, A., Broxvall, M., Gritti, M., LeBlanc, K., Lundh, R., Rashid, J., Seo, B., and Cho, Y. (2008). The peis-ecology project: Vision and results. In Intelligent Robots and Systems, 2008. IROS 2008. IEEE/RSJ International Conference on, pages 2329-2335.

[37] Salton, G., Wong, A., and Yang, C. (1975). A vector space model for automatic indexing. Communications of the ACM, 18(11):613-620

[38] Schade, S. (2010). Ontology-Driven Translation of Geospatial Data, volume 1 of GISDISS. Akademische Verlagsgesellschaft AKA GmbH, Heidelberg, Germany.

[39] Scheider, S. (2012). Grounding geographic information in perceptual operations, volume 244 of Frontiers in Artificial Intelligence and Applications. IOS Press, Amsterdam, The Netherlands.

[40] Scheider, S., Janowicz, K., and Kuhn, W. (2009). Grounding geographic categories in the meaningful environment. In Hornsby, K., Claramunt, C., Denis, M., and Ligozat, G., editors, Spatial Information Theory: 9th International Conference, COSIT 2009, Aber Wrac'h, France, September 2009, Proceedings, volume 5756 of Lecture Notes in Computer Science, pages 69-87, Heidelberg, Germany. Springer.

[41] Scheider, S., Probst, F., and Janowicz, K. (2010). Constructing Bodies and their Qualities from Observations. In Galton, A. and Mizoguchi, R., editor, Formal Ontology in Information Systems Proceedings of the Sixth International Conference (FOIS 2010), volume 209 of Frontiers in Artificial Intelligence and Applications, pages 131-144, Amsterdam, The Netherlands. IOS Press.

[42] Shaw, R., Turvey, M., and Mace, W. (1982). Ecological psychology: The consequence of a commitment to realism. In Weimer, W. and Palermo, D., editors, Cognition and the Symbolic Processes, volume 2, pages 159-226. Lawrence Erlbaum, Hillsdale, NJ.

[43] Stevens, S. (1946). On the theory of scales of measurement. Science, 103(2684):677-680.

[44] Stoffregen, T. (2003). Affordances as properties of the animalenvironment system. Ecological Psychology, 15(2):115-134.

[45] Turvey, M. (1992). Affordances and prospective control: An outline of the ontology. Ecological Psychology, 4(3):173-187.

[46] Warren, W. (1984). Perceiving affordances: Visual guidance of stair climbing. Journal of Experimental Psychology: Human Perception and Performance, 10(5):683-703.

[47] Wong, S., Ziarko, W., and Wong, P. (1985). Generalized vector spaces model in information retrieval. In Proceedings of the 
8th annual international ACM SIGIR conference on Research and development in information retrieval, SIGIR '85, pages 18-25, New York, NY. ACM.

[48] Zadeh, L. (1978). Fuzzy sets as a basis for a theory of possibility* 1. Fuzzy sets and systems, 1(1):3-28.

[49] Zadeh, L. (2001). A new direction in AI: Toward a computational theory of perceptions. AI magazine, 22(1):73-84.

[50] Zadeh, L. (2002). Toward a perception-based theory of probabilistic reasoning with imprecise probabilities. Journal of statistical planning and inference, 105(1):233-264.

[51] Zadeh, L. (2004). A note on web intelligence, world knowledge and fuzzy logic. Data \& Knowledge Engineering, 50(3):291-304

\section{Appendix}

The Appendix provides two example queries as they are used to retrieve necessary information to establish the transformation matrix.

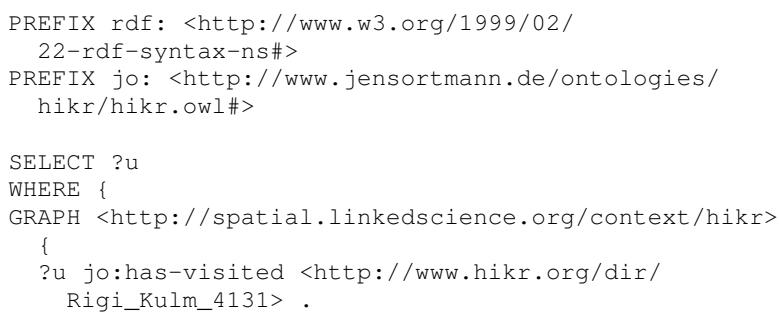

Listing 1: SPARQL Query for Step 1, finding users that have hiked paths that include the Rigi Kulm Waypoint.

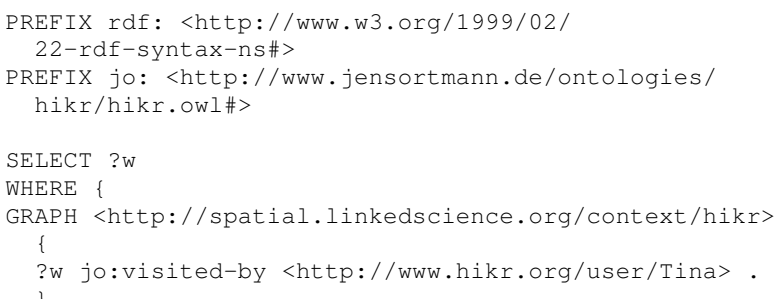

Listing 2: Example SPARQL Query for Step 2. Find the waypoints that the user Tina has visited. 